\title{
Narrativa
}

\section{Maschilità e letteratura: uno sguardo dagli USA}

\section{Anna De Biasio}

\section{(2) OpenEdition}

\section{Journals}

\section{Edizione digitale}

URL: https://journals.openedition.org/narrativa/524

DOI: $10.4000 /$ narrativa.524

ISSN: 2804-1224

\section{Editore}

Presses universitaires de Paris Nanterre

\section{Edizione cartacea}

Data di pubblicazione: 1 décembre 2018

Paginazione: 27-36

ISBN: 978-2-84016-325-1

ISSN: 1166-3243

\section{Notizia bibliografica digitale}

Anna De Biasio, «Maschilità e letteratura: uno sguardo dagli USA», Narrativa [Online], 40 | 2018, online dal 01 novembre 2021, consultato il 08 décembre 2021. URL: http://journals.openedition.org/ narrativa/524; DOI: https://doi.org/10.4000/narrativa.524

Narrativa est mise à disposition selon les termes de la Licence Creative Commons Attribution 4.0 International. 


\section{Maschilità e letteratura: uno sguardo dagli USA}

\section{RIASSUNTO}

Il saggio prende le mosse da una riflessione sulla genesi dei masculinity studies in area statunitense, per poi focalizzarsi sull'intersezione tra lo studio del maschile e la letteratura. Vengono quindi esaminati i principali approcci al discorso letterario attraverso alcuni esempi di studiosi influenti. Infine, viene preso in considerazione il caso di Henry James e la sua evoluzione nel panorama della critica, un'evoluzione che permette di mettere a fuoco possibili punti di forza e limiti dello studio della maschilità applicato al testo letterario.

\section{RÉSUMÉ}

Partant d'une réflexion sur la genèse des études sur les masculinités aux ÉtatsUnis, l'étude se concentre sur l'intersection entre les études sur le masculin et la littérature. Les principales approches au discours littéraires y sont analysées, par le biais d'exemples tirés des chercheurs les plus influents. Enfin, la chercheuse prend en considération le cas d'Henry James et son évolution dans le panorama de la critique littéraire, une évolution qui permet d'illustrer les points de force mais aussi les limites des études sur les masculinités utilisées pour le texte littéraire.

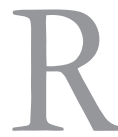

iflettere su identità maschile e letteratura significa porsi in una prospettiva fortemente interdisciplinare, in cui la storia e i generi letterari, così come le questioni relative a temi, struttura e stile, si trovano a essere contaminati - spesso assorbiti - da discorsi che attengono variamente alla sociologia, alla psicoanalisi, all'antropologia, all'economia, alle neuroscienze, e ad altro ancora. Un recente studio statunitense sulla figura del cornuto nella letteratura comica rinascimentale è in questo senso esemplare. Ispirandosi tanto a teorici come Lacan, Foucault, Althusser, quanto a studiose di gender studies come Judith Butler e Eve Kosofsky Sedgwick, l'autore intende la maschilità come "scopic drive, set of relations, mode of surveillance, 
corporeal and linguistic performance, and manipulation of a visual, spatial and imaginary social domain"; una costruzione sociale polimorfa che, sulla base di una nozione vertiginosamente ampia di testualità, si svilupperebbe "within and as the intertextual practices of a particularly written tradition, stretching from the earliest legal compendia, to canon law texts, to penance manuals, to criminal registers, to didactic clerical manuals, and finally to the comic narrative literature of the Middle Ages and Renaissance". . Gli Stati Uniti sono il luogo in cui la riflessione accademica sul maschile è maggiormente consolidata, al punto che nel 2019 la Stony Brook University (parte della State University di New York) inaugurerà un corso di laurea interamente dedicato ai masculinity studies $^{2}$. Ripercorrere alcune scansioni di tale riflessione, pertanto, offre spunti utili a ragionare sui punti forza e insieme sui possibili limiti delle sue intersezioni con il discorso letterario.

Un primo aspetto da considerare è la natura intimamente contraddittoria delle spinte che hanno portato lo studio sul maschile a emergere come sottocampo autonomo nella galassia dei gender studies. I men's studies non erano paradossalmente sempre esistiti, la storia, la scienza, l'arte, la letteratura essendo di fatto un'emanazione dell'operato maschile? Nelle fasi iniziali della battaglia femminista, l'uomo era visto infatti come il principale fautore di quell'ordine patriarcale che il movimento combatteva strenuamente, il responsabile della riduzione al silenzio della metà del genere umano che ora pretendeva di essere ascoltata. Prendere la parola, conquistare un ruolo e una visibilità pubblica significava più o meno implicitamente mettere a tacere chi aveva da sempre dominato la produzione dei discorsi sotto la fuorviante egida dell'universale. Proprio la sensibilizzazione operata dal femminismo nei confronti della vittimizzazione delle donne stimola tuttavia un'inedita attenzione da parte degli stessi uomini nei confronti del proprio ruolo nel mantenimento dell'oppressione di genere, un'attenzione tipicamente rappresentata nella sua fase nascente dai movimenti di autocoscienza maschile costituitisi negli anni Settanta ${ }^{3}$. Non solo la denuncia delle ingiustizie perpetrate contro le donne, ma la crescente conquista da parte di queste ultime di spazi e posizioni al di fuori dell'ambito

1. LA GuARDiA David, Intertextual Masculinity in French Renaissance Literature: Rabelais, Brantôme, and the Cent Nouvelles, London, Routledge, 2016, p. 6.

2. Presso la Stony Brook University era già presente un Center for the Study of Men and Masculinity, fondato e diretto da Michael Kimmel (cfr. Bennett Jessica, "A Master's Degree Program in...Masculinity Studies?", The New York Times, 8 agosto 2015).

3. Per una ricostruzione più approfondita della genesi dei masculinity studies rimando al mio saggio "Studiare il maschile", Allegoria, n 61, 2010, pp. 9-36. 
domestico contribuiscono a diffondere la percezione che la maschilità tradizionale sia in crisi. In sé ambigua, la nozione di crisi si accompagna a reazioni polarizzate, che da un lato puntano a correggere le storture del sistema patriarcale ripensando il maschile in un'ottica più rispettosa delle differenze (quella di genere ma anche quella sessuale, come chiede il Gay Liberation Movement sorto alla fine degli anni Sessanta), mentre dall'altro lato insistono sulla necessità di ri-mascolinizzare l'uomo contemporaneo, indebolito dagli effetti del femminismo e dagli eccessi della società tecnologica ${ }^{4}$.

I primi importanti studi che cominciano ad apparire nella seconda metà degli anni Ottanta prendono le mosse da questa messa in discussione dell'identità maschile, dimostrando di avere assimilato la lezione del femminismo là dove questo aveva rivendicato la necessità di trattare il genere come costruzione sociale e culturale e non come un aprioristico dato di natura. Robert Connell, Robert Seidler e Michael Kimmel sono unanimi nel considerare i valori del controllo e dell'autonomia, attributi per eccellenza del maschile, come il risultato di processi storici e dei condizionamenti culturali, mentre prende sempre più campo l'idea - tipico portato di una società multiculturale come quella americana, tendenzialmente organizzata in gruppi identitari - che esistano vari tipi di maschilità ${ }^{5}$ La classe, la razza, l'orientamento sessuale, l'età, sono tutte condizioni identitarie che tagliano trasversalmente il genere e che, combinandosi tra loro, riproducono a turno rapporti di dominio e subordinazione: la maschilità bianca, ad esempio, tende a "femminilizzare" quella nera o di altre etnie, così come quella gay. Un'altra idea forte che si afferma è l'esistenza, in una data società, di una forma egemonica di maschilità, che in virtù della sua possibilità di essere percepita e trasmessa come ideale culturale (ad esempio incarnandosi in personaggi fittizi o in attori cinematografici)

4. Sui movimenti maschili, cfr. Newton Judith, "Masculinity Studies: The Longed for Profeminist Movement for Academic Men?", in Gardiner J.K., Masculinity Studies and Feminist Theory, New York, Columbia University Press, 2002, pp. 176-192. Cfr. anche Adams Rachel, SAVRAN David, Introduction, in The Masculinities Studies Reader, Oxford, Blackwell, 2002, pp. 1-20.

5. Cfr. Connell Robert W., Masculinities, Berkeley-Los Angeles, University of California Press, 1992 (cfr. anche il precedente ID., Gender and Power: Society, the Person, and Sexual Politics); SEIDLER Victor J., Rediscovering Masculinity: Sexuality, Reason, Language, 1989; Kimmel Michael S., Messner Michael A., "Introduction", in Kimmel Michael S., Messner Michael A. (a cura di), Men's Lives, Boston-London, Allyn and Bacon, 1989, pp. xi-xxii. 
aumenta enormemente la propria capacità d’influenza, saldandosi con il potere reale amministrato dagli uomini ${ }^{6}$.

Gli studi concordano perciò nel configurare il maschile come un modello o un'aspirazione più che come un'identità stabile. Un ideale che, allo scopo di apparire come una realtà "naturalizzata", deve essere sottoposto a una costante convalida, o, come vuole una teoria influente di Judith Butler, a performance ripetute di genere, senza però che vi sia la possibilità di renderlo perfettamente aderente ai soggetti ${ }^{7}$. Ricorre spesso l'immagine della maschilità come banco di prova della capacità di assumere il potere (più che una forma effettiva di potere), come spinta alla leadership dominata dalla paura, dal senso di inadeguatezza e dal fantasma del fallimento, e ancora, nei lavori più legati alle categorie della psicanalisi, come forma di mancanza e "vuoto a essere". Un altro tratto ricorrente è il ricorso alla letteratura sia in quanto luogo privilegiato della costruzione dei soggetti maschili nella loro evoluzione storica, sia come terreno di applicazione della teoria. I lavori di Michael Kimmel, Kaja Silverman e Eve Kosovsky Sedgwick sono ottimi esempi delle linee di tendenza più rappresentative dei masculinity studies nel loro rapporto con lo specifico letterario.

Sociologo di formazione ed esponente della prima generazione di uomini ad essersi apertamente e consapevolmente confrontati con il femminismo della cosiddetta seconda ondata, Kimmel è autore capostipite di una tipologia di indagini ad ampio raggio in chiave di storia culturale, come si evince dal suo volume più noto, Manhood in America (1996). Per Kimmel, che vedel'orientamento maschile al dominio come il frutto del timore di essere dominati dagli altri, la letteratura è una risorsa utile a ricostruire le immagini dominanti della maschilità in un dato periodo, una tra le tante fonti di un vasto archivio che allinea sullo stesso piano romanzi, racconti, fumetti, manuali di pedagogia, regolamenti di associazioni, pamphlet politici, e ancora testi musicali, film e programmi televisivi. L'uso del testo letterario, che Kimmel stesso definisce parte di un approccio "eclettico" alle proprie fonti, è dunque dichiaratamente strumentale ${ }^{8}$. Benché soprattutto nei lavori più recenti l'autore dimostri un'ammirevole padronanza del dibattito letterario in tema di gender, il suo metodo argomentativo si basa su una lettura tutta contenutistica dell'opera, in cui tipicamente viene assegnato un valore ideologico all'intreccio, i personaggi sono esaminati in

6. Connell Robert W., Masculinties, cit., p. 77.

7. ButLer Judith, Gender Trouble: Feminism and the Subversion of Identity, New York-London, Routledge, 1990.

8. Kimmel Michael, Manhood in America: A Cultural History, New York, The Free Press, 1996, p. 10. 
quanto incarnazione di principi astratti o di particolari temi funzionali a sostenere una tesi e il testo stesso diventa un contenitore di passi che si prestano a illustrare gli argomenti in questione. In Manhood in America, ad esempio, la successione dei tipi dominanti della maschilità statunitense secondo Kimmel dal Genteel Patriarch allo Heroic Artisan, al Self-Made Man - viene identificata in nuce in The Contrast (1797) di Royall Tyler, un'opera teatrale postrivoluzionaria i cui personaggi maschili sono visti come lo specchio trasparente di tali forme identitarie esemplari'.

L'interesse di Kimmel per i modelli egemonici del maschile è tipico delle indagini di carattere sociologico o storico (si veda il fondamentale lavoro di Anthony Rotundo), così come del fiorente settore dei masculinity studies applicati al genere Western ${ }^{10}$. In Male Subjectivity at the Margins, Kaja Silverman si concentra invece sulle maschilità marginali facendo leva su strumenti teorici, in particolare sulla psicoanalisi. Specializzata in storia dell'arte e in teoria critica, Silverman attacca sin dalle premesse ciò che definisce la "fiction dominante", ovvero la credenza nell'egemonia maschile e nell'unità della famiglia eteronormativa, a sua volta basata sulla persuasione che fallo e pene coincidano. L'operazione di smantellamento della logica fallocentrica schiude la via alla messa in luce di maschilità devianti, potenzialmente resistenti al potere, esplorate grazie a una rilettura di Althusser, Foucault, e soprattutto dei modelli psicoanalitici di Freud (in particolare la normalizzazione edipica) e di Lacan. A Silverman interessa esaminare la soggettività maschile là dove essa si apre al femminile, accogliendo in sé la castrazione, l'alterità, la specularità, il masochismo: tutti tropi che il maschile convenzionale denega programmaticamente al fine di potersi costituire in identità. Se i materiali su cui l'autrice lavora sono principalmente il cinema e la letteratura, per quanto riguarda quest'ultima gli autori prescelti sono Henry James, Lawrence d'Arabia e Marcel Proust. I testi sono discussi in profondità, con una speciale attenzione ai ruoli/ribaltamenti di potere agiti dai personaggi in relazione al tema della sessualità, e tuttavia è evidente che l'interesse letterario è subordinato per Silverman a quello teorico. James, Lawrence e Proust sono oggetti di speculazione (che non include però l'analisi formale del testo) "because their work provides such fertile terrain for the exploration of a

9. Ibid., pp. 13-17.

10. Rotundo Anthony E., American Manhood: Transformations in Masculinity from the Revolution to the Modern Era, New York, Basic Books, 1993. Tra i primi studi sui legami tra maschilità e Western, ora numerosissimi, cfr. Mitchell Lee Clark, Westerns: Making the Man in Fiction and Film, Chicago, University of Chicago Press, 1996 e Tompkins Jane, West of Everything: The Inner Life of Westerns, New York, Oxford University Press, 1992. 
form of subjectivity which, because it assumes a directly textual guise, is unusually amenable to theoratical analysis, i.e. authorial subjectivity": un tipo di autorità testuale che si presta all'analisi teorica proprio in quanto si rivelerebbe, contrariamente alle apparenze, capace di sovvertire la maschilità normativa ${ }^{11}$.

Ugualmente ancorato a un imponente apparato teorico (Foucault, Freud, Lévi-Strauss, Gayle Rubin, René Girard) e ugualmente centrato sul problema della sessualità, il lavoro della docente di letteratura inglese Eve Kosofsky Sedgwick differisce dall'impostazione di Silverman sotto due importanti punti di vista. Innanzitutto, mentre Silverman insegue le devianze del maschile sul piano dell'inconscio letterario, Sedgwick si concentra sul legame tra maschilità dominante e marginale laddove tale legame è paradossalmente tanto negato quanto esibito. Nello studio che contribuirà in maniera decisiva allo sviluppo dei Queer Studies, Between Men, la studiosa analizza uno spaccato di letteratura inglese dal Seicento all'Ottocento, rilevando nei testi la presenza di speciali legami tra maschi ${ }^{12}$. Benché ufficialmente la maggior parte di tali testi tratti di storie d'amore tra uomini e donne, queste ultime sono in realtà funzionali (in base alla classica economia di scambio del sistema patriarcale descritta da Lévi-Strauss) a mediare particolari rapporti tra gli stessi uomini. Grazie a una rilettura dei triangoli amorosi studiati da René Girard, il legame tra uomo e uomo, che può variamente esprimersi nella forma della rivalità ma anche dell'amicizia, del cameratismo, della paideia, appare ovunque intessuto di desiderio: un desiderio che come tale si nutre di eros, benché non necessariamente assuma una valenza sessuale (o genitale). Sedgwick mostra così l'esistenza di una continuità tra omosocialità e omosessualità, due manifestazioni di un'unica formazione ininterrotta che nella nostra epoca viene radicalmente denegata ('emergere dell'omofobia contemporanea, sotto forma di "panico omosessuale" a fine Ottocento, viene approfondito nel successivo Epistemology of the Closet, 1990). A differenza di Silverman, che presuppone l'azione di fantasie devianti inconsce, Sedgwick rinviene nelle strategie rappresentative messe in campo nei testi l'omosessualità latente negli alvei del potere maschile. L'altro punto di grande distanza da Silverman (ma anche da Kimmel e in generale dalla maggior parte dei masculinity studies applicati alla letteratura) è infatti costituito dal metodo

11. Silverman Kaja, Male Subjectivity at the Margins, New York-London, Routledge, 1992, p. 11.

12. Sedgwick Kosofsky Eve, Between Men: English Literature and Male Homosocial Desire, New York, Columbia University Press, 1985. Nati dalla risignificazione positiva di un insulto rivolto agli omosessuali ("queer"), i Queer Studies si propongono di indagare le identità e le sessualità non normative. 
dell'argomentazione, la quale, pure essendo originata nella teoria, viene messa alla prova attraverso un close reading del testo letterario. Strutture sintattiche e ritmiche (nel caso della poesia), stringhe semantiche, figure retoriche e altri aspetti strutturali e stilistici sono parte integrante della costruzione della tesi. Particolarmente degna di nota, sia per la sua audacia sia per il clamore accademico che suscitò negli anni Novanta, è l'affermazione che l'erotismo anale che pervade l'opera di Henry James sia riprodotto a livello della struttura della frase, ad esempio nel romanzo The Ambassadors, la cui convenzionale sequenza di soggetto-verbo-oggetto appare turbata (ma anche "squarciata") dall'insinuazione di sempre nuove proposizioni o espressioni qualificanti ${ }^{13}$.

Come suggeriscono queste rapide note, nei masculinity studies che si occupano di letteratura è molto frequente il riferimento a Henry James, la cui evoluzione come oggetto di critica letteraria testimonia più di altri il travolgente impatto che gli studi culturali hanno avuto su quest'ultima come campo di sapere specifico $^{14}$. Considerato un writers' writer e un Maestro della forma dagli stessi contemporanei, James è stato per più di mezzo secolo un autore-chiave delle scuole critiche interessate a porre in primo piano aspetti stilistici e tecnici dell'opera letteraria, dal Modernismo al New Criticism, fino allo strutturalismo. Questioni di punto di vista e voce narrante, metodo ("drammatico" vs. "pittorico"), struttura della prosa e genere letterario (su tutti il romanzo, di cui James è stato un grande teorico) hanno a lungo dominato l'arena interpretativa, spesso restituendo un'immagine dello scrittore come elitario stilista rinchiuso nella torre d'avorio della forma narrativa ${ }^{15}$. A partire dagli anni Settanta, con l'avvento della cosiddetta "cultura del dissenso" e di paradigmi critici volti a schiudere il testo a molteplici contesti, l'interesse per il James formalista è gradualmente

13. "[...] the typifying sentence of The Ambassadors, whose relatively conventional subject-verb-object armature is disrupted, if never quite ruptured, as the sac of the sentence gets distended by the insinuation of one more, and just one more, and another, another, and impossibly one more, qualifying phrase or clause"; SEDGWICK Kosofsky Eve, "Is the Rectum Straight? Identification and Identity in The Wings of the Dove”, in Ead., Tendencies, Durham, Duke University Press, 1993, p. 102.

14. Di Henry James si occupa anche Kimmel in relazione alla crisi dell'identità maschile di fine Ottocento. Cfr. Kimmel Michael, History of Men: Essays on the History of American and British Masculinities, Albany, SUNY Press, 2005, pp. 45-46.

15. Tra i primi studi di taglio formalista, cfr. LubBock Percy, The Craft of Fiction, New York, C. Scribner's Sons, 1921. La critica su Henry James è vastissima e stratificata e non avrebbe senso darne conto parziale qui. Per un bilancio e una ricca rassegna bibliografica, cfr. Izzo Donatella, "Introduction: The Manifold Arts of Re-vision", in EAD., Portraying the Lady: Technologies of Gender in the Short Stories of Henry James, Lincoln, University of Nebraska Press, 2001, pp. 3-20. 
tramontato per cedere il passo a esplorazioni dell'opera jamesiana nei suoi vari rapporti con la filosofia, le questioni di classe, l'industria editoriale, la cultura di massa, le minoranze etniche, il genere sessuale, e altro ancora ${ }^{16}$. Nel rigoglioso ambito della critica jamesiana a tema gender, l'attenzione all'identità maschile occupa una fetta consistente, rivelatasi in grado di illuminare e intrecciare tra loro dimensioni che spaziano dalla biografia alla storia letteraria, dalla storia sociale ed economica ai contenuti di romanzi e racconti.

Non più confinata al repertorio dei giudizi di scarsa virilità (l'autore fu alternativamente stigmatizzato dai contemporanei e successivamente come "eunuco", "femminuccia", "Henrietta"), la problematica della maschilità di e in James è stata affrontata in tutta la sua poliedrica complessità. Si è riconosciuta la piena consapevolezza da parte dello scrittore della propria incapacità di aderire all'ideale maschile borghese dell'Ottocento, negli USA rigidamente centrato sugli attributi di vigore fisico, eterosessualità (comprovata dal matrimonio con prole), aggressività negli affari e in generale affermazione di sé nella sfera pubblica: tutte caratteristiche che, quando appaiono incarnate da qualche personaggio fittizio (Christopher Newman in The American, Basil Ransom in The Bostonians, il dottor Sloper in Washington Square), sono oggetto di una sottile critica da parte del testo jamesiano, che ne mette in luce i limiti e le contraddizioni. Si è altresì messo in luce come James, lungi dall'occupare passivamente la propria posizione identitaria non normativa, difendesse attivamente la scelta della vita artistica, rivendicando per il romanzo uno spazio di prestigio nel campo letterario, capace di "(ri)maschilizzare" il genere. Per attuare quest'opera di distinzione, l'autore prese spesso le distanze dalle donne che affollavano il campo della scrittura romanzesca (e con le quali condivideva gli argomenti principali delle opere, storie di donne e intrighi sociali legati a corteggiamenti e matrimoni), dissciando i fini artistici del romanzo dagli scopi di intrattenimento commerciale attribuiti a tanta produzione femminile ${ }^{17}$.

Un'ampia porzione degli interventi critici ruota attorno al problema della sessualità jamesiana, di difficile definizione data l'assenza di prove circa l'omosessualità dell'autore, ma fonte di fascinazione per la presenza - nei testi creativi e non - di una ricchissima rete di allusioni omoerotiche. Il 2003 ha visto la pubblicazione di due importanti monografie sul tema, che riflettono alcuni dei

16. Ibid., p. 3.

17. Per una bibliografia parziale degli studi su James e il maschile, con riferimento alle tesi qui esposte, cfr. Haralson Eric, Johnson Kendall, Critical Companion to Henry James: A Literary Reference to his Life and Work, New York, Infobase Publishing, 2009, pp. 414-416. 
pregi e difetti dei masculinity studies come lente attraverso cui guardare la letteratura ${ }^{18}$. In Henry James and the Suspense of Masculinity, Leland Person sostiene che l'opera dell'autore suggerisce la possibilità che genere e sessualità siano fluidi, passibili di decostruzione e di improvvisazione; in particolare, il posizionamento soggettivo che James distribuisce tra i suoi personaggi maschili identificherebbe "contested sites at which 'sex, gender, sexual practice, and desire', to use [Judith] Butler's terms, come together in a state of dynamic tension". In Henry James and Queer Modernity, Eric Haralson esplora l'elusiva associazione tra "queer" e "omosessuale", un legame semantico che andava consolidandosi negli anni dell'attività creativa di James e che proprio attraverso le sue sottili codificazioni della maschilità non normativa avrebbe influenzato l'opera di Willa Cather, Ernest Hemingway e Gertrude Stein.

Se Person si focalizza quasi esclusivamente sull'opera di James, Haralson apre l'interrogazione ad altri autori e rafforza l'impostazione storicista che condivide con Person (entrambi fanno riferimento alla confusione tassonomica nelle categorie di sesso e genere a cavallo tra Otto e Novecento), inseguendo le trasformazioni contestuali subite dal concetto di queerness. Nei due casi, tuttavia, il metodo analitico-argomentativo è simile. Pareri critici dell'epoca, scholarship jamesiana, fonti primarie letterarie ed extra-letterarie (come saggi e corrispondenza) si avvicendano come oggetto dell'analisi senza che siano poste differenze statutarie tra tipologie testuali, e soprattutto senza che il discorso letterario sia riconosciuto come codice specifico, fondato su elementi come strutture narrative e tecniche della rappresentazione aventi a loro volta alle spalle una storia di teorizzazioni e interpretazioni. Nell'Introduzione, Person afferma che ricostruire gli esperimenti jamesiani con sesso e genere "means looking closely at James's own richly metaphorical language as a metadiscourse on gender and sexuality" "19; ma nel prosieguo dell'argomentazione il riferimento alle metafore - e in generale alla tessitura stilistica del testo - è sporadico e occasionale, e ciò che prevale è una lettura ravvicinata dell'intreccio, dei profili dei personaggi e dei loro rapporti, dei dialoghi, di alcuni aspetti lessicali. Allo stesso modo, benché la lettura di Haralson sia più marcatamente "semiotica", centrata com'è sulla disamina di una varietà di "significanti (proto)gay", in realtà anche in questo caso ciò che prevale nella discussione è il grado di distanza dei

18. Person Leland S., Henry James and the Suspense of Masculinity, Philadelphia, University of Pennsylvania Press, 2003; Haralson Eric, Henry James and Queer Modernity, Cambridge, Cambridge University Press, 2003.

19. Person Leland S., Henry James and the Suspense of Masculinity, cit., p. 18. 
vari personaggi, o di altri aspetti dell'opera, dai modelli ideali o tipi dominanti del maschile (ad es.: "[...] The Europeans flirts with a type of masculinity at variance with normative designs and desiderata" $" 20$ ).

Nella cosiddetta "anglosfera", il discorso sulle identità rinvenibile nei testi letterari è sentito tuttora come cogente, un correttivo all'oblio a cui le precedenti visioni della letteratura come quella idealistica, stilistico-filologica, persino la marxista avevano consegnato particolari soggetti e gruppi sociali: presenze vive che rivendicano non solo visibilità e rappresentazione, ma anche l'importanza di interpellare sia l'atto creativo sia quello critico come gesti situati, originati da particolari condizioni identitarie che celano di volta in volta intricati rapporti di potere. L'interrogazione del maschile è in questo senso un'occasione preziosa per portare alla luce il fatto che anche l'identità più resistente all'oggettivazione è in realtà il frutto di una fabbricazione basata su meccanismi di esclusione $\mathrm{e}$ distinzione (dal femminile, da forme di maschilità considerate indesiderabili o fallimentari), un tentativo di recisione dei legami di interdipendenza che lascia zone grigie, crepe e ibridazioni tutte da indagare. Allo stesso tempo, ciò che suggerisce il caso della critica jamesiana degli ultimi decenni è che a fronte di inedite aperture di senso, la letteratura come oggetto di critica testuale può risultare fortemente appiattita, così come un intero patrimonio di precedenti riflessioni sulle forme dei testi prestarsi a essere troppo facilmente liquidato. Discipline tradizionalmente più impervie al discorso identitario (come l'italianistica) potrebbero in questo caso godere di un vantaggio competitivo. Aprendosi con ritardo allo studio del genere - e in particolare del maschile potrebbero dimostrare che quest'ultimo non solo non è incompatibile con una sensibilità verso i procedimenti della rappresentazione, ma che i più validi strumenti di decostruzione dell'ideologia delle opere possono essere proprio lo stile, $i$ tropi, i dispositivi testuali che servono a codificarle.

Anna De Biasio

Università di Bergamo

20. Haralson Eric, Henry James and Queer Modernity, cit., p. 51. 


\title{
I vestiti perduti dell'imperatore. \\ La nuova nudità politica del maschile di fine millennio
}

\begin{abstract}
RIASSUNTO
L'articolo tratta della rappresentazione mediatica del corpo maschile, nell'Italia a cavallo del millennio, contestualizzandola storicamente in una più generale trasformazione delle relazioni di genere nella contemporaneità. A una nuova "nudità" ora gli uomini, avendo perduto il privilegio dell'invisibilità, non possono evitare di sottostare; si configura così una nuova condizione maschile, nella quale i tradizionali travestimenti retorici della virilità non sono più efficaci, come in passato, a occultare le contraddizioni di un modello identitario sempre più anacronistico, all'indomani della doppia rivoluzione del neocapitalismo e del neofemminismo.
\end{abstract}

\section{RÉSUMÉ}

L'article se concentre sur la représentation médiatique du corps masculin en Italie, au tournant du $\mathrm{XXI}^{\mathrm{e}}$ siècle, en les encadrant dans une transformation plus générale des relations de genre à l'époque contemporaine. Le privilège de l'invisibilité perdu, les hommes ne peuvent plus éviter de se soumettre à une nouvelle "nudité", qui devient ainsi une nouvelle condition masculine. À la suite de la double révolution du néocapitalisme et du néoféminisme, les déguisements rhétoriques traditionnels de la virilité ne permettent plus, comme dans le passé, d'occulter les contradictions d'un modèle identitaire de plus en plus anachronique.

\section{IL DECLINO DELL'INVISIBILITÀ MASCHILE}

Sul piano interpretativo, l'ambito in cui questa riflessione vorrebbe iscriversi è quello delle immagini, degli stereotipi, delle rappresentazioni diffuse della corporeità maschile nell'Italia di fine Novecento; una dimensione, questa, da contestualizzare a sua volta all'interno della più ampia cornice dell'evoluzione storica delle identità, delle relazioni e dei ruoli di genere nel corso degli stessi decenni. Particolare attenzione dedicherò qui ai linguaggi mediatici, che 
assumono proprio fra anni ottanta e novanta una funzione normativa e pedagogica, anche riguardo alla cultura diffusa di genere, molto più incisiva che nel passato.

Da questa prospettiva analitica, la novità più significativa del periodo considerato mi sembra quella di una emersione dall'invisibilità della mascolinità in quanto configurazione identitaria sessuata: negli ultimi due-tre decenni del Novecento, in sintesi, si moltiplicano nella comunicazione pubblica i riferimenti espliciti agli uomini in quanto nomini. L'invisibilità è da sempre privilegio del potere, ovviamente non soltanto in una dimensione di genere; molto spesso, storicamente, un soggetto dominante siè preteso impronunciabile, irriconoscibile nella sua specificità, e quindi anche, in una certa misura, politicamente inattaccabile. È un procedimento che Roland Barthes, a proposito dell'ascesa storica della borghesia come classe dominante, ha chiamato e-nominazione: "la borghesia ha cancellato il suo nome [...] la borghesia si definisce come la classe sociale che non vuole essere nominata".

Nel caso dell'invisibilità maschile (dell'uomo "normale": eterosessuale, bianco, più spesso borghese), è questo un processo da inquadrare entro la più ampia dinamica novecentesca per cui il genere dominante ha visto progressivamente erosa, decennio dopo decennio, la propria potestà tradizionale di affermarsi come soggetto neutro e universale. La maggiore esposizione cui è sottoposto nei linguaggi mediatici il corpo maschile alla fine del millennio, spesso in forme inedite, si configura quindi anche come un indicatore cruciale di questo grande mutamento politico. I tradizionali codici della neutralità, che sin dal tardo Ottocento avevano occultato la parzialità maschile - e dunque anche il corpo sessuato maschile - per porre al riparo la supremazia degli uomini dai venti sovversivi dell'egualitarismo, sembrano ora diventare sempre più inefficaci e anacronistici.

Per buona parte del ventesimo secolo, in estrema sintesi ${ }^{2}$, la partita patriarcale si è giocata anche e soprattutto sul rilancio di un energico virilismo come orizzonte primario delle identità e relazioni di genere: quindi, sull'esaltazione a tutti i livelli della maestà sociale maschile, sulla denigrazione misogina, sulla discriminazione e persecuzione della "devianza" omosessuale. All'interno di tale scenario, la finzione di naturalità e trascendenza della supremazia maschile è

1. Barthes Roland, Miti d'oggi, Torino, Einaudi, 1974 [ed. or. Mythologies, Paris Édition du Seuil, 1970], pp. 218-219. Corsivo nel testo.

2. Rimando, per più ampie considerazioni nel merito, al mio L'invenzione della virilità. Politica e immaginario maschile nell'Italia contemporanea, Roma, Carocci, 2011. 
stato un caposaldo simbolico della sua legittimazione, come - specularmente doveva apparire naturale l'inferiorità delle donne e l'insanità dei "pervertiti". Ma nella seconda parte del secolo l'impalcatura retorica virilista iniziava a crollare sotto i colpi consecutivi della secolarizzazione neocapitalistica, che sin dagli anni sessanta ha identificato l'austerità patriarcale e certe concezioni rigidamente misogine come ostacoli all'espansione dei beni di consumo, e della demolizione neofemminista dell'ordine virilista, al punto che negli anni settanta il virilismo stesso ha toccato il suo minimo storico in termini di legittimazione diffusa.

A partire da questa fase, decade progressivamente anche la funzionalità delle coperture cognitive che avevano più o meno efficacemente occultato la parzialità sessuata maschile, connotando il maschile stesso come un ossimorico genere universale; emerge così una sempre più chiara nudità degli uomini in quanto maschi, ora spogliati dei loro paramenti regali di assolutezza, neutralità, imparzialità. Come nella favola di Andersen, adesso il re è nudo, lo è agli occhi di chiunque e in modo apparentemente irreversibile.

D'altro canto, non sono mancate e non mancano neppure oggi le tenaci insistenze (in verità, non proprio trascurabili) di chi continua ad aggrapparsi saldamente alla vecchia invisibilità maschile; dopo tutto, in effetti, gli uomini rimangono tuttora e sempre il genere del comando. Nella dimensione simbolica legata al mondo militare, ad esempio, che non è certo divenuta residuale di decennio in decennio, si celebra propriamente il livellamento delle individualità all'interno di un corpo collettivo, di una massa in uniforme: e l'uniforme è uno dei veicoli grazie ai quali la realtà concreta di corpi maschili differenti e differentemente vulnerabili si sublima in un'astratta forza collettiva, rivestendosi provvidenzialmente di virilità grazie alla più classica missione guerriera ${ }^{3} . \mathrm{Nel}$ mondo del business e della grande politica internazionale, peraltro, si continua a celebrare una storica standardizzazione in direzione di quello stile corporeo maschile che lo studioso di moda Ted Polhemus, non a caso, definisce come Uomo invisibile:

Dall'età del colonialismo fino ai nostri giorni, per farsi prendere sul serio sulla scena mondiale, un uomo d'affari o un leader politico devono prima rinunciare

3. Aspesi Natalia, "Miles gloriosus. Il ritorno delle uniformi militari nell'immaginario maschile", in MALOSsI Giannino (a cura di), Uomo oggetto. Mitologie, spettacolo e mode della maschilità, Bergamo, Bolis, 2000, pp. 148-153. 
alla propria fisicità e nascondere il corpo dietro l'uniforme, oggi universale, dell'Uomo invisibile: un anonimo completo di cattivo taglio ${ }^{4}$.

Con il collasso tardo-novecentesco del virilismo classico, insomma, il panorama identitario maschile si è fatto straordinariamente frastagliato. Così, nelle culture degli uomini del terzo millennio vecchie strategie, inediti compromessi e nuove rotture più o meno polemiche convivono, si sovrappongono e confliggono; al contempo, in prospettiva storica appare tuttavia evidentissimo il mutamento identitario maschile nel segno di un netto declino - graduale e relativo se vogliamo, ma reale - della tradizionale invisibilità sessuata. Si tratta, senza dubbio, di un mutamento epocale nei rapporti di genere.

È anche necessario considerare, d'altra parte, come tali trasformazioni si iscrivano entro una più generale tendenza culturale alla "corporeizzazione" del sé, secondo certa sociologia, in quanto esasperazione della dimensione fisica nel tentativo di dare stabilità a un'identità individuale sempre più precaria e faticosa: il corpo è ormai il campo di un "progetto", ma anche, e sempre più con la fine del millennio, uno "strumento di contrattazione" sul terreno dell'identità personale. Secondo Federico Boni, in particolare, si tratterebbe di una dinamica culturale complessiva che tende alla "piena auto-regolazione individualizzata del corpo".

\section{EMERSIONI SESSUATE}

Negli ultimi anni del Novecento, in effetti, si registra nella comunicazione di massa (non solo italiana, certamente) una vera e propria affermazione iconografica del corpo maschile, anche nel senso di un'inedita presenza del nudo maschile. Non è certo un caso che una simile nudità maschile emerga dopo il già citato collasso del virilismo classico, e forse pure per tale ragione questa epifania presenta un carattere politico fortemente ambivalente: da un lato costituisce una sorta di abbandono forzato della tradizionale invisibilità, e quindi si configura come una sorta di corto circuito oggettivo dei classici processi virilisti di riproduzione patriarcale; dall'altro, è questo anche un estremo tentativo di riafferma-

4. Polhemus Ted, "L'uomo invisibile", in Malossi Giannino (a cura di), Uomo oggetto, cit., p. 47.

5. Cfr. Giddens Anthony, Modernity and Self-Identity. Self and Society in the Late Modern Age, Cambridge, Polity Press, 1991.

6. Boni Federico, Men's help. Sociologia dei periodici maschili, Roma, Meltemi, 2004, p. 12, anche per la citazione successiva. 
zione di identità di genere nette e polarizzate, a voler scongiurare proprio quella confusione che è da sempre considerata nemica mortale della logica virilista stessa. Quasi che, a fronte di una linea di demarcazione sempre più labile fra generi dicotomicamente intesi, non rimanga altra soluzione che affidarsi sempre più a quello che tipicamente viene considerato il "grado zero" delle identità sessuate, il corpo nudo; nudo, "naturale" e dunque "vero" nel suo essere inconfondibilmente maschile o femminile.

Già nei primi anni novanta, certe novità della rappresentazione mediatica dell'uomo appaiono piuttosto evidenti. Ugo Volli, all'inizio di quel decennio, registra infatti una importante trasformazione quanto alla esponibilità del corpo maschile: il nudo virile inizia ad apparire sistematicamente nell'iconografia pubblicitaria ${ }^{7}$. Anche alla luce di quanto accennato più sopra, un "re nudo" è innanzitutto un sovrano la cui mascolinità sia inevitabilmente visibile, e quindi problematizzabile e discutibile: un simile denudamento è dunque, di fatto, una specie di emersione dalla clandestinità degli uomini in quanto genere. Si tratta probabilmente del massimo grado di vulnerabilità identitaria che il genere maschile abbia raggiunto nella storia recente delle relazioni di genere; e dunque, letteralmente, di una nudità politica a tutto tondo.

Di fatto, l’impossibilità di ricorrere ai tradizionali travestimenti di una neutralità quasi metafisica rende la mascolinità suscettibile - nella propria finitezza politica, nella propria concretezza anche fisiologica - di osservazione e valutazione dall'esterno, conferendo un potere inedito al giudizio delle donne sugli uomini stessi e sui loro corpi. È quindi anche sul piano dell'emersione di uno sguardo desiderante femminile, ora in parte legittimato (ma con mille ambivalenze) nel senso comune diffuso, che si può misurare il mutamento dei rapporti di potere fra i generi negli ultimi decenni del millennio. Si afferma, propriamente, un nuovo regime di visibilità maschile eterosessuale: tendenzialmente, $\mathrm{i}$ corpi degli uomini non possono più evitare di esporsi allo sguardo e al desiderio femminile.

Ma la nuova vulnerabilità maschile (o meglio: la decadenza dei paraventi politici che da lungo tempo la nascondevano), sul finire del Novecento, risente anche di trasformazioni e tensioni che ovviamente non hanno origine solo in una dimensione culturale, o comunque tutta interna alla sfera simbolica del genere in senso stretto. Negli stessi anni, ad esempio, cioè fra il decennio ottanta e novanta, si compie in Occidente la parabola epocale della deindustrializzazione:

7. Cfr. Volli Ugo, "Un corpo violento e artificiale", in ButAzzi Grazietta et alii (a cura di), Virilità e trasgressione, Novara, Istituto Geografico De Agostini, 1992. 
è la fine definitiva del fordismo, e con esso della lunga stagione gloriosa del breadwinner, del capofamiglia maschio come primario o esclusivo procacciatore di reddito. Fenomeni quali la precarietà occupazionale, la stessa disoccupazione ormai cronica, la dequalificazione professionale, tutti massicciamente diffusi a partire da questo periodo, riducono ai minimi termini i margini di riproduzione di quel ruolo sociale che da più di un secolo rappresentava uno dei fondamenti della mascolinità tradizionale.

C'è in particolare un'opera cinematografica che realizza narrativamente, e quasi in presa diretta, una mirabile sintesi di tutto ciò. Prodotta nel 1997, la commedia britannica Full Monty mette in scena prima gli effetti sessuati della fine della città-fabbrica sulle vite concrete di alcuni personaggi maschili, poi la loro angosciata percezione di un mutamento femminile radicale (una mutazione genetica, la definiscono gli attoniti personaggi), infine la decisione economicamente necessitata, per questi ex operai squattrinati, di farsi oggetto dello sguardo desiderante delle donne. Ma soprattutto l'opera di Peter Cattaneo descrive minuziosamente gli effetti della faticosa scelta della nudità sull'autorappresentazione dei protagonisti in quanto uomini, che dunque esperiscono una vera e propria "scoperta" del proprio corpo e della sua vulnerabilità a fronte dello scrutinio delle donne.

Nel film, insomma, uomini "normali" si ritrovano inaspettatamente in una posizione che è stata storicamente esclusiva del genere femminile, quella di doversi sottomettere a una sorta di tribunale dell'altro genere che giudicherà il corpo esaminandolo nei più intimi dettagli (lo strip di Full monty è appunto integrale: l'esposizione dei genitali è l'unica specialità che sentono di poter offrire questi uomini fisicamente non straordinari). Anche sul piano narrativo, in questo caso l'accettazione di un denudamento totale implica quindi a un tempo la rinuncia forzata al privilegio dell'invisibilità di genere (persino in riferimento al proprio stesso sguardo su di sé), il riconoscimento di una potestà giudicante femminile che incide immediatamente sulla propria autostima in quanto maschi, e infine la condivisione fra maschi di un'acuta e dolorosa sensazione di vulnerabilità sessuata.

\section{LA NUDITÀ COME CRISI}

Quanto la nuova visibilità del maschile abbia conseguenze problematiche sul benessere identitario degli uomini stessi, lo testimonia anche la diffusione a livello mediatico di rappresentazioni pessimistiche, sconfortanti, ovvero immediatamente apocalittiche sulla condizione attuale del cosiddetto sesso 
forte. Un elemento molto rilevante di questo scenario complessivo è ad esempio lo sviluppo, sempre negli anni novanta, di un discorso pubblico (ancora una volta, mediatico in particolare) ed esplicito sulla cosiddetta crisi della mascolinità. Si tratta di un tema non proprio inedito - se ne parlava già ampiamente in tutta Europa alla fine dell'Ottocento -, ma che si presenta come un motivo retorico davvero dilagante a partire dagli anni settanta e ottanta del Novecento. È senz'altro interessante notare che, peraltro, il declino dell'invisibilità maschile è stato interpretato come causa ed effetto, a un tempo, di questa percezione diffusa di "crisi". Secondo Marco Alberio,

con l'ingresso del maschio, del corpo maschile e soprattutto della sua sensualizzazione sulla scena mediatica ha preso piede quella che potremmo definire la retorica della crisi maschile [...]. Il concetto della crisi del maschio ha cominciato ad essere un tema ricorrente soprattutto da quando l'uomo e la sua fisicità sono diventati oggetto molto utilizzato nelle rappresentazioni mediatiche. Quindi, è ipotizzabile che questo tema sia fortemente legato alla comunicazione e alla maggiore e nuova visibilità del genere maschile ${ }^{8}$.

Anche per Boni, analogamente,

al discorso sulle "maschilità in crisi" si lega [...] il presunto senso di disagio provocato per le "maschilità egemoni" dall'essere diventate oggetto e non soggetto dell'occhio altrui [...] infatti, la corporeità maschile [...] diviene un problema per le modalità con cui diviene oggetto di consumo ${ }^{9}$.

Dalla fine del millennio siamo entrati ormai chiaramente, aggiunge Boni, in una fase in cui "allo sguardo del 'maschio sulla femmina' si è affiancato quello del 'maschio sul maschio' (oltre a quello della 'femmina sul maschio'), rendendolo socialmente accettabile"10. Compare insomma sulla scena delle identità sessuate, e riceve l'importante legittimazione del Mercato, quello che Tim Edwards studioso britannico del consumo maschile - ha definito "l'uomo allo specchio"11. Il ruolo di garanzia identitaria che svolge in tale ambito la comunicazione pubblicitaria, all'interno di una società che inizia sempre più a delegare al Mercato stesso la ristrutturazione (per non dire la definizione normativa in

8. Alberio Marco, "La metamorfosi del corpo maschile nei media", in Ruspini Elisabetta (a cura di), Uomini e corpi. Una riflessione sui rivestimenti della mascolinità, Milano, Franco Angeli, 2009, p. 215.

9. Boni Federico, Men's help, cit., p. 29. Corsivi nel testo.

10. Ibid., p. 31.

11. Edwards Tim, Men in the Mirror: Men's Fashion, Masculinity, and Consumer Society, London, Bloomsbury, 2016. 
senso stretto) non solo degli assetti politici complessivi, ma anche degli ingredienti simbolici con cui gli individui sono chiamati ad assemblare le proprie identità sessuate in quanto stile, non va in alcun modo sottovalutato. Come ancora osserva Alberio,

si è passati da una fase in cui il corpo maschile (nella sua esposizione) era assente nel testo mediatico ad un'altra in cui la sua presenza è sempre più costante e in aumento. Questo è essenzialmente dovuto al fatto che gli uomini hanno iniziato ad essere percepiti come un importante target di mercato per moltissimi prodotti, non solo quelli a loro tradizionalmente indirizzati: automobili, sigarette, alcolici e rasoi ${ }^{12}$.

Non può dirsi certo nuovo l'utilizzo di una figura maschile come protagonista del messaggio pubblicitario; in qualità di esperto, ad esempio, un uomo compariva spesso come garante del prodotto sin dagli albori della moderna pubblicità televisiva. Ma in quel caso il suo corpo era ben coperto dagli abiti che ne indicavano il ruolo professionale, e che, mentre lo nascondevano allo sguardo, costituivano gli indicatori più importanti dell'autorità virile. Alla fine del Novecento, invece, il mutamento è da questo punto di vista davvero rilevante:

Fino alla metà degli anni Novanta, periodo che coincide con il massimo sviluppo delle TV commerciali, è raro osservare nelle pubblicità italiane e nei programmi televisivi, corpi maschili integralmente o parzialmente esposti. Anche quando si pubblicizzavano prodotti per il corpo e la cura maschile (rasoi, schiume da barba o al massimo profumi) il corpo veniva raramente mostrato nudo. Il più delle volte era coperto da maglietta intima e accappatoio, come mostra ad esempio lo spot Gillette Dopo Barba Contour del 1982 $2^{13}$.

Ma il copione mediatico della "crisi del maschio" non si limita a un generico denudamento: molto più insidiosamente, giunge a investire in modo frontale quello che classicamente è considerato il vero nucleo identitario del vero uomo. Presentato come "testo" autentico dell'identità sessuata degli uomini, il corpo maschile è infatti attraversato mediaticamente, nell'Italia degli anni novanta, anche da tensioni che ne mettono radicalmente in discussione gli attributi più delicati. Ben prima del boom commerciale del Viagra (apparso sul mercato mondiale nel 1998), il tema della "crisi" maschile si àncora dunque alla sfera della sessualità in modo davvero drammatico; la perdita di potenza subìta dalla

12. Alberio Marco, "La metamorfosi del corpo maschile nei media", cit., p. 215.

13. Ibid., p. 220. 
millenaria legittimazione del potere maschile si traduce anche in un'immagine di impotenza fisicamente e impietosamente connotata, fin negli anfratti più imbarazzanti della virilità. Nel nuovo regime della corporeità manifesta, ma anche grazie a una nuova e robusta tendenza cultural-commerciale alla medicalizzazione delle tensioni identitarie socialmente diffuse, un simile senso di non-potere degli uomini, originato in una dimensione più globalmente politica, sfocia insomma anche in una dolorosa diagnosi (letteralmente) clinica destinata a coinvolgere, o addirittura a minacciare, tanto il singolo uomo quanto il genere maschile nel suo complesso ${ }^{14}$.

Così, ad esempio, intorno alla metà degli anni novanta periodici italiani molto diffusi dedicano spazi importanti alle disfunzioni erettili, fenomeno, a quanto si sostiene, in preoccupante aumento: "Sesso debole. Uomini in crisi, un italiano su dieci soffre di impotenza", titola una copertina del Venerdi di Repubblica; "Ciao maschi. Problemi sessuali in aumento", annuncia il supplemento sui temi della salute dello stesso quotidiano; "Provaci ancora uomo. Dossier/Salvare la virilità. Tutto quello che avreste voluto sapere sul sesso ex forte ma non avete mai osato chiedere", recita la titolazione di copertina dell'Espresso. Non si può evitare di registrare come questo avvilente scenario proponga in primo piano, iconograficamente, una molto inquietante controparte sessuata: in alcune di tali immagini infatti compaiono ora un'enorme mano femminile nel gesto di aprire gli slip del povero maschio per scrutarne il contenuto, ora una giovane donna molto divertita mentre punta lo sguardo verso il piccolo pene di un classico Bronzo.

Negli stessi anni, varie altre narrazioni mediatiche investono le intimità maggiormente salienti del corpo maschile, insistendo sul più delicato dei simboli della virilità: in un'analisi della comunicazione pubblicitaria del 1997, ad esempio, si registrava un crescente ricorso non solo a una corporeizzazione, ma precisamente a una genitaližazione del maschile nella pubblicità visiva:

La mascolinità, meglio la virilità, comincia ad essere sottolineata fisicamente fino alla provocazione; la serie di inguini nudi di Benetton piuttosto che la sequenza di membri ben individuabili nelle diverse posizioni, ancorché inguainati in un paio di jeans di "Energie", costituiscono uno stacco rispetto ad una tradizione nella quale la virilità non veniva mai rafforzata attraverso

14. Cfr. Ferrero Camoletto Raffaella, Bertone Chiara, "Introduzione: La medicalizzazione della sessualità maschile: prospettive di analisi e campi di ricerca", in EAD. (a cura di), Le fragilità del sesso forte. Come medicalizzare la maschilità, Milano-Udine, Mimesis, 2016. Per una prospettiva storica più ampia, cfr. inoltre McLAREN Angus, Storia dell'impotenza, Bologna Odoya, 2009 [ed. or. 2007]. 
la dimostrazione genitale, ma lasciata ad una generica prestanza fisica o agli atteggiamenti sociali ${ }^{15}$.

\section{La “Rivoluzione amorale” del Mercato}

Sono tutti esempi, questi, propri di una dimensione della comunicazione direttamente funzionale al consumo. A ben vedere, il nuovo marketing sessuato di fine millennio, mentre trasmette messaggi che sempre più esplicitamente chiamano in causa - per esaltarli, in teoria - i più cari attributi virili, in realtà rappresenta una sconsacrazione tombale della virilità: l'esposizione reiterata del corpo maschile, sottolineando ripetutamente la parzialità sessuata degli uomini, per ciò stesso tende a svestire di assolutezza metafisica la virilità medesima e quindi conduce a un fatidico svelamento della sua vulnerabilità. Da un lato insomma questo vergognoso segreto di Achille finisce per essere, di fatto, sbandierato ai quattro venti; dall'altro, l'espansione di nuovi settori economici legati alla mascolinità come target colloca la stessa identità sessuata degli uomini all'interno diuna vetrina pubblica ormai del tutto incompatibile, tendenzialmente, con lo storico privilegio patriarcale dell'invisibilità di genere.

Se a fine millennio può accadere tutto questo è certamente, come ho già accennato, perché nei decenni precedenti il virilismo come ordine retorico "normale" è stato demolito da una radicale contestazione neofemminista, che ne ha svelato alla radice tutta la natura ideologica: la rivoluzione politica e culturale compiuta dai movimenti delle donne, sin dagli anni sessanta del Novecento, ha infatti definitivamente strappato il genere maschile dal piedistallo di una supremazia indiscussa, strappando al contempo il velo delle gravi contraddizioni intrinseche all'equilibrio identitario tradizionale degli uomini (i quali, senza un saldo ancoraggio alla posizione del comando, accusano immediatamente una sorta di sindrome debilitante della mascolinità: una logica politica destinata fatalmente al tracollo, a fronte dell'affermazione - almeno formale - del principio di uguaglianza).

Ma questo "svelamento" della mascolinità, alla fine del millennio, si compie anche perché il mercato ha finito per individuare nell'invisibilità maschile un limite importante all'espansione sessuata dei beni di consumo; ancora una volta,

15. Caligaris Gianni, "Ostento dunque sono? Il maschio di fine millennio nell'immaginario pubblicitario", "Alfazeta", 63-64 (1997), Derive del maschile. Gli uomini dopo il femminismo, p. 86. 
quindi (come già, lo si è accennato, negli anni sessanta ${ }^{16}$ ), gli interessi "profani”" dell'economia prevalgono impietosamente sulle ultime velleità di sacralità e trascendenza del virilismo. Ha scritto nel 2000 Giannino Malossi:

Il tiro di cui viene fatto oggetto il maschio, sotto forma di obiezioni e messa in discussione della identità di genere e rappresentazione della perplessità maschile contemporanea, è oggi il gioco più frequente nella comunicazione mediatica e nella moda, che di questa è la versione per così dire reificata, trasformata in $\operatorname{merci}^{17}$.

Tuttavia, per fare della mascolinità un vero e proprio target, non è sufficiente che essa venga sconsacrata, secolarizzata, e dunque - nella logica del vecchio statuto patriarcale dell'invisibilità - depotenziata: l'uomo in quanto uomo deve anche, ben più radicalmente, essere messo a fuoco, esibito, interrogato. 亡̀ letteralmente una vivisezione in pubblico, cadenzata sulle esigenze supreme del Mercato; un vero e proprio sacrilegio che non può non configurarsi come l'ultima profanazione della virilità. Siamo qui di fronte, probabilmente (e come altre volte nella storia), a una sorta di rivoluzione amorale: laddove il Mercato, pur perseguendo una strategia moralmente indifferente, cioè essendo tendenzialmente indisponibile a piegarsi a ragioni che le proprie interne finalità economiche non contemplino, produce però di fatto un mutamento politico sui generis, ma reale e profondo, spingendo verso una secolarizzazione oggettiva delle identità e delle relazioni di genere.

Analogamente, le superiori esigenze aziendali non si fanno alcuno scrupolo di contribuire, con la loro potenza mediatica, a un'evoluzione della rappresentazione maschile che concretamente sfocia in una moltiplicazione delle incertezze identitarie degli uomini, se ciò appare funzionale agli interessi produttivi e commerciali prevalenti. Si pensi soltanto, a tale proposito, allo sviluppo impressionante che nuovi consumi legati al corpo, e anche per questo necessariamente sessuati (cosmetici, palestre e centri fitness, integratori alimentari, chirurgia estetica), hanno conosciuto a cavallo del millennio.

Un fenomeno di massa, a ben vedere, che in un angosciante circolo vizioso non può che esporre il genere maschile a un'ulteriore visibilità, davvero inedita per profondità e latitudine sociale. È chiaro, infatti, che tali esperienze corporee

16. Cfr. Bellassai Sandro, "Mascolinità, mutamento, merce. Crisi dell'identità maschile nell'Italia del boom", in CAPuzzo Paolo (a cura di) Genere, generazione e consumi. L'Italia degli anni Sessanta, Roma, Carocci, 2003.

17. Malossi Giannino, "Uomo oggetto", in ID. (a cura di), Uomo oggetto, cit., p. 27. 
si configurano come in se stesse ansiogene, e lo diventano doppiamente nel caso degli uomini: perché spesso sono legate a un senso di inadeguatezza personale e virile, ma anche perché appartengono a una dimensione di cura di sé che è classicamente associata al femminile. L'effetto è allora quello di alimentare sul piano sociale la paurosa sensazione di una confusione crescente tra i generi, e dunque di produrre nuovi timori di svirilizzazione, in un vortice ansiogeno davvero drammatico per l'identità maschile.

Alcune strategie commerciali paiono in effetti guardare precisamente, e piuttosto avidamente, a questo orizzonte di "sessuazione angosciosa". All'inizio del millennio - nel 2000, per l'esattezza - esordisce anche in Italia il fenomeno delle riviste maschili: fa da apripista "Men's Health", edizione italiana di un magazine statunitense, che nella patria del Latin lover vende la bellezza di 400.000 copie al mese $^{18}$. Vari altri periodici analoghi sono apparsi sulla scena successivamente, a comporre un coro comunicativo e pedagogico che si concentra in modo a dir poco maniacale sul corpo, sulla dieta, sulla potenza fisica e sessuale e su semplici ricette per raggiungerla con poca fatica: si tratta in effetti di un vero e proprio Men's help, secondo il titolo del già citato saggio di Boni dedicato a questi nuovi media. Gli esiti a cui giunge una simile narrazione ansiogena possono anche essere paradossali; ecco ad esempio come "Fox Uomo" parla degli inestetismi del petto maschile, e dei modi di risolverli:

La ginecomastia (termine scientifico che indica l'eccessivo ingrandimento delle mammelle nell'uomo) può causare insicurezza e difficoltà nei rapporti con le persone, soprattutto con le donne. Il problema del "seno" non è tipico solo degli uomini in sovrappeso o di mezza età, ma riguarda anche i trentenni attenti all’alimentazione e con una vita dinamica. E quando né la dieta né la ginnastica risolvono il problema, se vuoi proprio esibire un torace asciutto e tonico al punto giusto, puoi prendere in considerazione l'intervento chirurgico ${ }^{19}$.

Il titolo di questo articolo è straordinariamente interessante, ai fini del nostro discorso: "Re di coppe? No, grazie". La sequenza della storica detronizzazione maschile si fa qui davvero micidiale. Da re, a re nudo, a re di coppe: comprensibilmente, è davvero troppo per gli ultimi orfani della famosa virilità italica.

Sandro BeLLASSAI Università di Bologna

18. Cfr. Boni Federico, Men's help, cit., p. 41.

19. Re di coppe? No, grazie, "Fox Uomo", maggio 2003, p. 61. 


\title{
Le seduzioni pericolose dell'nomo a cavallo. Mussolini, Gadda, Palazzeschi e la cultura virile in Italia tra primo novecento e fascismo
}

\begin{abstract}
Riassunto
Il saggio si sofferma sul mito della virilità nell'uomo a cavallo, considerandolo qui in particolare attraverso la figura di Mussolini condottiero. A partire dalle concezioni del maschile e del femminile elaborate da Otto Weininger e abbracciate in Italia da Giovanni Papini agli inizi del Novecento, e appoggiandosi anche alle teorie del tempo sulla folla e sull'educazione avanzate da Gustave Le Bon, l'autrice analizza il mito del Duce a cavallo sviluppatosi in Italia nel periodo fascista, guardando anche a come reagirono a questa figura iconica due scrittori molto diversi tra loro come Carlo Emilio Gadda e Aldo Palazzeschi.

\section{RÉSUMÉ}

Cet article se concentre sur le mythe de la virilité du cavalier, en l'analysant par le biais de l'image de Mussolini chevalier. L'autrice part des théories du masculin et du féminin élaborées par Otto Weininger et reprises en Italie par Giovanni Papini au début du $\mathrm{Xx}^{\mathrm{e}}$ siècle et s'appuie ensuite sur les théories de l'époque sur la foule et l'éducation proposées par Gustave Le Bon. Elle analyse ainsi le mythe du Duce à cheval, qui s'est développé en Italie à la période fasciste, sans négliger la réaction à cette image de deux écrivains très différents : Gadda et Aldo Palazzeschi.
\end{abstract}

\section{VIRILITÀ DELL’UOMO A CAVALLO}

In una celebre scena del film di Ettore Scola Una giornata particolare (1977), ambientato durante il fascismo e precisamente a Roma il 6 maggio 1938, Marcello Mastroianni, che interpreta Gabriele, un ex radiocronista omosessuale, sfoglia un album di fotografie del Duce messo insieme da Antonietta, la casalinga dirimpettaia impersonata da Sophia Loren che gli sta preparando un caffè. "Bel cavallo, eh?", fa Mastroianni indicando una delle foto che ritrae il 
Duce in sella in uniforme militare. Antonietta riconosce subito Ned, uno degli esemplari della scuderia di Mussolini, e sciorina i nomi di altri due mostrando fieramente di conoscerli a memoria ed essere informatissima su tutto quanto riguarda il Capo del fascismo. Gabriele prova allora a mostrare il Duce cavaliere sotto un'altra luce; ma Antonietta non lo segue su questo e anzi ricorda il giorno in cui ha incontrato "a tu per tu" Mussolini a cavallo rimanendone letteralmente folgorata:

- [G.] Si dice che ogni mattina spezzi le reni di un cavallo e la sera quelle di una donna. Poveri cavalli. E povere donne!

- [A.] Se ne dicono tante su di lui. [...] Lo sapete che io una volta quattro anni fa l'ho incontrato? A tu per tu.

- [G.] Davvero? E dove?

- [A.] A Villa Borghese. Lui passava a cavallo, io mi fermai e lui al galoppo mi lanciò uno sguardo. Diventai tutta una fiamma. Stavo con le borse della spesa, figuratevi. Mi sentii le gambe spezzate, poi mi cominciò a girare, a girare, a girare tutt'intorno e... caddi a terra svenuta.

- [G.] Con uno sguardo... lanciato... al galoppo?

- [A.] Eh... Alcuni passanti mi aiutarono, e come Dio volle raggiunsi la circolare. Quello stesso giorno scoprii che ero incinta di Vittorio'.

La scena restituisce in maniera esemplare l'aura che in epoca fascista circondava la figura del Duce cavaliere, vera e propria icona di virilità che si credeva dotata addirittura di poteri magici (qui, nella fantasia della donna, di una potentia seminandi esercitata con il solo sguardo).

Mussolini a cavallo piaceva così tanto alle donne? Pare di sì. Claretta Petacci, che ne era da tempo diventata l'amante, lo descriveva così nel suo diario il 26 febbraio 1938, dopo averlo ammirato anche lei al galoppatoio di villa Borghese: "bellissimo, abbronzato, forte sul cavallo bianco. [...] divino, grande come un Dio possente, splendido di forma"2. Un'altra donna, Wera B.M., gli inviò il 14

1. Una giornata particolare (1977), regia di Ettore Scola, versione restaurata dall'Associazione Philip Morris Progetto Cinema, DVD, Surf Film, s.d., da 55:40 a 57:36.

2. Petacci Claretta, Mussolini segreto. Diari 1932-1938, a cura di Mauro Suttora, Milano, Rizzoli, 2009, p. 221. 
febbraio 1935 da Reggio Emilia una poesia intitolata Dux, composta pochi mesi prima, datata 28 ottobre 1934, che esordiva così:

A cavallo, imponente: eretto, fiero, il volto maschio, da romano antico sculto nel bronzo; balenanti gli occhi, grandi, imperiosi, da trascinatore... ${ }^{3}$

Qui siamo sul Gianicolo, non a Villa Borghese, ma la reazione dell'io poetico femminile non è molto diversa da quella delle spettatrici del galoppatoio romano: "Io immobil stava, muta, affascinata / a la solenne, altissima visione"

Wera B.M. e Claretta Petacci non dovevano essere sole nel loro entusiasmo per il Duce cavaliere, se è vero quanto scriveva con esasperato livore Carlo Emilio Gadda in Eros e Priapo alla fine della dittatura: "Le femine [...] trovarono ch'egli era il mastio de' mastii" perché "elle perdono addirittura le staffe se quello le isguarda e le concupisce ha tra le gambe un cavallo". Ma è davvero così, come scrive Gadda, che "la donna ama e sogna il militare a cavallo, il cojonello a cavallo, il tenente e il cavallerizzo a cavallo" perché "gli sproni e' fanno un suggerimento crudele di quella inesorabile pressura che il mastio su di lei esercita"s?

Tra gli scrittori che hanno descritto con maggiore efficacia e sottigliezza questa fascinazione per il militare in sella è stato Aldo Palazzeschi. In Sorelle Materassi (1934), raccontando delle due ricamatrici affacciate alla finestra ad ammirare il passaggio dei soldati di cavalleria, Palazzeschi scrive:

Ma lo spettacolo più emozionante era costituito dal passaggio di un reggimento di cavalleria o di artiglieria [...]; ragazzoni sicuri, virili, piantati bene, lenti nei movimenti, parchi di gesti, anche quelli che rivelavano origine campagnola esercitavano sulle donne un potere possente. [...] [Teresa] Guardava estasiata un colonnello e non meno di un capitano in piena maturità, ma in tale rigoglio virile da farle abbacinare gli occhi con uno sguardo solamente. Quella solidità e sicurezza, quell'aria di comando e di salute l'attraevano dolcemente $[\ldots]$. Carolina invece $[\ldots]$ non poteva tollerare la vista di quegli omaccioni sanguigni,

3. Bontтi Giorgio (a cura di), Caro Duce. Lettere di donne italiane a Mussolini, 19221943, prefazione di Camilla Cederna, Milano, Rizzoli, 1989, p. 110. Sono i vv. 1-4.

4. Ibid., p. 111. Sono i vv. 36-37.

5. GadDa Carlo Emilio, Eros e Priapo. Versione originale [1944-45], a cura di Paola Italia e Giorgio Pinotti, Milano, Adelphi, 2016, p. 45. 
dall'aspetto spregiudicato e brutale, e che mostravano, stringendo la groppa del cavallo, delle coscie enormi, massicce come colonne, e un'aria di sufficienza e di autorità, ma soprattutto di sazietà e destrezza da farle correre un brivido di terrore sotto la pelle ${ }^{6}$.

Qui il maschio a cavallo provoca sulla femmina un'attrazione dolce oppure un brivido di terrore che è anche piacere, ma Palazzeschi ci presenta un intero reggimento, tanti militari di fatto indistinti, non un unico cavaliere. La moltiplicazione di questi "ragazzoni sicuri, virili" verso cui si orientano gli sguardi di Teresa e Carolina fa da contraltare alla repressione della femminilità delle due ricamatrici, che si sono rinchiuse in un mondo dove l'Eros non ha spazio. I contorni di questa parata militare poi si allargano ulteriormente: di questa virilità a buon mercato, di cui si può godere osservandola in sicurezza dalle finestre o dal lato della strada, si fanno spettatrici insieme alle due Materassi anche tutte le altre donne lì intorno, in una celebrazione del maschio che finisce per essere perfino gioiosa nella sua indistinta generosità:

E siccome alle sorelle [Materassi] si univano donne e fanciulle del casamento, i soldati passando lanciavano occhiate scherzose e voraci, provocando i sorrisi, le grida e le risate delle fanciulle, le occhiate calme e soddisfatte delle spose mature, la giocondità delle vecchie; lanciavano complimenti, frizzi, parole calde o grassocce, saluti, o gettavano baci ridendo, mostrando la bianchezza dei denti, producendo uno scompiglio nel gruppo delle spettatrici, allegrezza e confusione; e con tale generosa abbondanza, propria della gioventù, da potersi illudere che di tanto ben di Dio ce ne fosse per tutte, anche per le brutte, anche per le vecchie?

Con Palazzeschi siamo di fronte a un meccanismo opposto a quello offerto dalla figura del Duce cavaliere. Mussolini a cavallo era sempre l'unico personaggio in sella: anche se accompagnato da altri cavalieri, si poneva in posizione avanzata e isolata rispetto agli altri e diventava l'unico su cui si concentrava l'attenzione di chi lo osservava. Inoltre Mussolini dalla sella non lanciava occhiate scherzose, complimenti, frizzi, parole calde o grassocce, e tanto meno baci, ma era percepito - lo si è già detto - "imponente, eretto, fiero" (Wera B.M.), "divino, grande come un Dio possente" (Claretta Petacci), folgorante al punto che il suo solo sguardo muto bastava a un concepimento (Antonietta).

6. Palazzeschi Aldo, Sorelle Materassi [1934], edizione 1934, a cura di Francesca Serra, Milano, Mondadori, 2001, pp. 61-62.

7. Ibid., pp. 62-63. 


\section{Maschio, Femmina e ANimale}

Nel sistema di valori dominanti di inizio Novecento i caratteri di maschile e femminile erano rigidamente distinti e contrapposti. Giovanni Papini nel suo libro Maschilità (1915) si rifà al dualismo presentato da Otto Weininger in Sesso e carattere (1903) e postula un "contrapposto iroso tra Maschio e Femmina, tra maschilità e femminilità". In uno dei saggi che compongono il libro, intitolato Miele e pietra (1910), Papini scrive:

sono weiningeriano. Ci sono i sessi spirituali e non solo i fisici. Quando parlo di maschio intendo, ora, la forza, l'energia, la durezza, la fierezza; quando parlo di femmina la mollezza, la dolcezza, la voluttuosità blanda, il tono minore, le lacrime facili, il pettegolìo spiritosetto e la musicalità svaniente ed estenuante?.

Papini istituisce delle opposizioni: "tra le armi e la donna, tra la patria e la donna, tra il dovere e la donna, tra lo spirito e la donna, tra l'aeroplano e la donna..." ${ }^{\prime 10}$. Sono opposizioni che entrano nella cultura nazionalista, futurista e poi fascista in Italia ${ }^{11}$; in particolare l'uomo nuovo del fascismo doveva incarnare forza, energia, durezza, fierezza e riconoscersi nelle armi, nella patria, nel dovere, nello spirito e nella fede ideale ${ }^{12}$; e tutti questi erano sentiti in opposizione alla donna.

Un'altra differenza fondamentale tra uomo e donna veniva posta nel diverso rapporto con l'istinto sessuale, la razionalità e l'inconscio. "La donna è continuamente sessuale, l'uomo solo a intermittenza. L'istinto sessuale in lei è sempre desto $[\ldots]$, mentre nell'uomo esso tace durante intervalli più o meno

8. Papini Giovanni, Maschilità [1915], Firenze, Vallecchi, 1921, p. 7.

9. Ibid., p. 95. Il saggio era stato pubblicato originariamente su La Voce, II, 35, 11 agosto 1910.

10. Ibid., p. 96.

11. Cfr. Mosse George L., L'immagine dell'uomo. Lo stereotipo maschile nell'epoca moderna, Torino, Einaudi, 1997, pp. 207-208.

12. Sull'uomo nuovo fascista la bibliografia è molto vasta. Tra i saggi più recenti si possono vedere quelli raccolti in BernhARD Patrick e KLINKHAMMer Lutz (a cura di), L'uomo nuovo del fascismo. La costruzione di un progetto totalitario, Roma, Viella, 2017, con relativa bibliografia. 
lunghi"13, scriveva Weininger. In conseguenza di questa diversa dipendenza dall'istinto sessuale, "L'uomo [...] vive coscientemente, la donna [...] inconsciamente"14.

In base a questo sistema di rigide contrapposizioni e a questa diversa relazione con l'inconscio, come si potevano disegnare i rapporti tra i sessi? Anche su questo Weininger era stato netto:

la donna si aspetta sempre dall'uomo la chiarificazione delle proprie idee oscure [...]. Là dove la donna non s'è fatta che rappresentazioni incerte senza ancora una chiara coscienza, essa aspetta, desidera ed anzi esige dall'uomo quell'articolazione del pensiero, che man mano si sviluppa nel di lui discorso [...] una donna sente come criterio della virilità la superiorità anche intellettuale dell'uomo, $[\ldots]$ essa si sente attratta irresistibilmente dall'uomo, il cui pensiero le si imponga $[\ldots]$. La donna [...] riceve la propria coscienza dall'uomo: per l'uomo-tipo di fronte alla donna-tipo, di cui è il complemento ideale, la funzione sessuale consiste appunto nel rendere cosciente l'incosciente ${ }^{15}$.

Anche in questo caso siamo ben lontani dalla prospettiva di un Palazzeschi: nelle Sorelle Materassi, lo abbiamo visto, gli uomini dei reggimenti a cavallo - "lo spettacolo più emozionante" - non impongono nessun pensiero razionale, non rappresentano nessuna forma di coscienza per Teresa e Carolina o per le loro vicine; anzi i cavalieri solleticano i sensi delle donne riaprendo la comunicazione tra i sessi a quel principio di piacere represso al massimo grado nelle vecchie ricamatrici, chiuse in una morale del lavoro sessualmente fin troppo castrante.

I rigidi termini del discorso di Weininger si ritrovano invece perfettamente nella caratterizzazione del rapporto tra il Duce a cavallo e la folla quale era proposto dalla propaganda fascista: Mussolini rappresentava il principio maschile razionale che regolava gli istinti di una folla di spettatori percepita come femminile quando non direttamente animale. Lo si vede bene per esempio nella descrizione che Mario Carli fa dell'inaugurazione dello Stadio Littoriale di

13. Weininger Otto, Sesso e carattere, traduzione integrale di Julius Evola di Geschlecht und Charakter (1903), nuova edizione riveduta, introduzione di Fausto Antonini, Roma, Edizioni Mediterranee, 1992, p. 134 (parte seconda, "I tipi sessuali"; cap. II, "Sessualità maschile e sessualità femminile").

14. Ibid., p. 146 (parte seconda, "I tipi sessuali”; cap. III, "Coscienza maschile e coscienza femminile”).

15. Ibid. 
Bologna nel suo romanzo L'Italiano di Mussolini (1930), quando il Duce si presenta a cavallo e parla alla folla riunita sugli spalti:

Quand'egli diceva alle moltitudini "Popolo, tu sei", esse sentivano [...] di sentirsi prese nella morsa di una volontà trascinante, che benedivano e adoravano come l'animalità dell'uomo benedice e adora il cervello che la plasma e la conduce. [...] Che significato aveva quell'entusiasmo oceanico per l'Uomo [...] dritto sul suo cavallo sauro [...]? [...] Non voleva forse dire tutta la gioia di aver trovato il Capo sicuro che, rivelandola a se stessa, le garantiva la nuova dignità, il nuovo splendore cui violentemente aspirava? ${ }^{16}$

Oppure, ancora, Mussolini a cavallo inteso come principio regolatore razionale di animali e uomini (o di uomini che sono come animali) lo troviamo in un opuscolo della collana "Mussolinia" dedicato a Mussolini e lo sport (1927), in cui Carlo Dall'Ongaro scrive a proposito dei cavalli del Duce:

Vi sono dei giorni in cui i cavalli sono nervosi così che riesce difficile governarli e insellarli. [...] Ma quando Mussolini, con agile mossa salta in sella e raccoglie le briglie, i nobili quadrupedi sembrano metter giudizio immediatamente. Come gli uomini, sentono di essere alla mercè di un essere contro il quale è inutile ribellarsi, [...] egli domina il cavallo così come domina le volontà umane dei singoli e delle masse: Con una energia logica, sicura, che non ammette contrasti, dubbiosità, eccezioni...

Il "dominatore" si rivela anche nei confronti dei cavalli come in quelli degli uomini! ${ }^{17}$

Dall'Ongaro è persino compiaciuto dalla similarità di uomini e animali di fronte alla volontà dominatrice e alla logica sicura del Duce.

In fondo era questa "funzione sessuale", per dirla ancora con Weininger, ciò contro cui si scagliava retrospettivamente Gadda in Eros e Priapo. Carli e Dall'Ongaro la salutavano con entusiasmo; Gadda la vituperava. Partendo anch'egli dal presupposto che "La moltitudine [...] la è femmina", attraverso la donna Gadda se la prendeva contro "L'io collettivo $[. .$.$] guidato [\ldots]$ molto più dagli 'istinti', cioè in definitiva da Eros, che non da ragione o da ragionata conoscenza" ${ }^{18}$. I suoi strali, se erano diretti in primis verso le donne, intendevano

16. Carli Mario, L'Italiano di Mussolini, Milano, Mondadori, 1930, pp. 197 e 200.

17. Dall'Ongaro Carlo, Mussolini e lo sport, "Mussolinia", periodiche mensili, fascicolo n. 33, Mantova, Edizioni Paladino, anno VI dell'era fascista [1927-28], p. 20. Il nome dell'autore compare a p. 37.

18. Gadda Carlo Emilio, Eros e Priapo, cit., pp. 15 e 30. 
poi colpire tutti quelli che avevano rinunciato all'esercizio del pensiero abbandonandosi a "Eros nelle sue forme inconscie e animalesche"19, quell'Eros stuzzicato dal Duce "issàtosi a bravazzare lassù a cavallo"20. Gadda notava infatti che "Simili alle femine [...] sono dimolti omini assetati di dogma, vogliosi non altro che prosternarsi a un enunciatore di dogmi, libidinosi ripeterne la formula" 21 finché "arrivasi a un punto nel quale" gli uomini sentono "l'aggregazione, "la pluralità', come una donna"22. Come una donna, o come un animale, potremmo anche dire sulla scia di Carli e Dall'Ongaro.

In tutto questo che parte aveva il cavallo? Contava veramente che Mussolini fosse ritto sulla sella mentre folgorava le donne che lo guardavano o parlava agli uomini femminilizzatisi/animalizzatisi nella massa?

\section{Mussolini e Gustave Le Bon}

La caratterizzazione della folla in senso femminile risale alla fine dell'Ottocento $^{23}$; la ritroviamo nei primi decenni del Novecento diffusa da autori di grande successo come Gustave Le Bon, autore della famosa Psychologie des foules (1895). In quest'opera Le Bon sostiene che "Les foules sont partout féminines" e che gli individui che le compongono sono caratterizzati da "évanouissement de la personnalité consciente, prédominance de la personnalité inconsciente", "n'étant capables ni de réflexion ni de raisonnement"

Come rapportarsi a soggetti di questo tipo, come comunicare con loro, come guidarli e influenzarli? Il libro di Le Bon intendeva esattamente rispondere a questi problemi. Poiché "les foules ne sont pas influençables par des raisonnements, et ne comprennent que de grossières associations d'idées" ${ }^{25}$, bisogna far leva su queste per agire sull'inconscio. Uno dei mezzi principali consigliati da Le Bon era la ripetizione, in base alla convinzione che "la chose répétée finit par s'incruster dans ces régions profondes de l'inconscient où s'élaborent les motifs de nos actions" ${ }^{\prime 26}$.

19. Ibid., p. 37.

20. Ibid., p. 22.

21. Ibid., p. 46.

22. Ibid., p. 73.

23. Cfr. Falasca-Zamponi Simonetta, Lo spettacolo del fascismo, Soveria Mannelli, Rubbettino, 2003, pp. 36-40.

24. Le Bon Gustave, Psychologie des foules, Paris, Alcan, 1895, p. 27, 20 e 55.

25. Ibid., p. 101.

26. Ibid., pp. 112-113. 
Mussolini si professava "un des plus fervents adeptes" ${ }^{27}$ del pensatore francese. "J'ai lu toute l'œuvre de Gustave Le Bon" 28 , dichiarò in un'intervista a Pierre Chanlaine nel 1926. Tra le opere di Le Bon, oltre alla Psychologie des foules, ve n'è anche una intitolata L'équitation actuelle et ses principes (1892), in cui si ritrovano gli stessi principî visti sopra, applicati però ai cavalli; essi valgono dunque per tutti gli esseri, umani o animali:

Par suite de la répétition, l'obéissance au langage du cavalier deviendra de moins en moins hésitante, de plus en plus instinctive. De nouveaux réflexes se formeront graduellement dans les centres nerveux de l'animal, et le jour où ils seront solidement fixés, l'exécution de l'ordre correspondant à un signe donné sera automatique. L’obéissance est alors absolue. [...] Qu'il s'agisse du cheval ou d'un être quelconque, y compris l'homme, l'éducation n'est véritablement complète que quand les associations sont devenues automatiques. Elles se passent alors dans les régions de l'inconscient et ne demandent plus aucun effort. [...] Toute éducation doit avoir pour but de transformer les actes conscients en actes inconscients ${ }^{29}$.

Mussolini amava andare a cavallo e lo faceva ogni mattina ${ }^{30}$. Le sue cavalcate non si limitavano però allo svago o all'attività sportiva e tanto meno alla sfera privata. Infatti ancor più che al galoppatoio di Villa Borghese o nel maneggio privato di Villa Torlonia, il duce a cavallo lo si poteva vedere in pubblico nelle occasioni ufficiali più importanti, come gli anniversari della Marcia su Roma o la rivista delle truppe; per non dire che la sua effigie di condottiero era riprodotta su una quantità e varietà innumerevole di supporti ${ }^{31}$. Tanta insistenza nel mostrarsi a cavallo e nelle vesti di condottiero evidentemente non era neutra: se l'equitazione era, secondo Le Bon, un'attività analoga a quella di controllare le folle, Mussolini presentandosi su un destriero non faceva che raddoppiare la sua attività di controllo inculcando riflessi automatici: nel cavallo si trattava di riflessi fisici che comportavano l'obbedienza ai comandi del cavaliere, secondo quanto avveniva in un normale addestramento in maneggio; negli uomini erano riflessi

27. Chanlaine Pierre, Mussolini parle, Paris, Tallandier, 1932, p. 61.

28. ID., "Une heure avec M. Mussolini”, in La Science et la Vie, 108, giugno 1926, p. 477.

29. LE BON Gustave, L'Équitation actuelle et ses principes. Recherches expérimentales, Paris, Firmin-Didot, 1892, pp. 133-134, 138-139.

30. Cfr. Dall'Ongaro Carlo, Mussolini e lo sport, cit., pp. 11 e 15 in particolare.

31. Su questo mi permetto di rimandare a SiCA Beatrice, "Il Duce e il popolocavallo: politica, pedagogia e propaganda nell'immagine di Mussolini condottiero", in Studi culturali, xv, 2, agosto 2018, pp. 159-188. Il presente articolo in effetti riprende e amplia un'analisi già iniziata in quella sede. 
ideologici, per così dire, che andavano a determinare un'obbedienza politica attraverso la riproposizione di slogan e coreografie in cui il Duce si imponeva come il Capo.

Insomma Mussolini, attento lettore di Le Bon, incantatore e agitatore di folle, seduttore, uomo di stato, cavallerizzo e condottiero offre un concentrato e una capillare messa in pratica delle teorie del tempo sul maschile e sul femminile, sulla psicologia individuale e collettiva e sui mezzi per affermare il potere del singolo sulle masse. Di fronte alle milizie o agli uomini e alle donne del popolo schierati di fronte a lui la posizione in sella moltiplicava l'effetto di virile dominio del Capo attraverso cui venivano inculcati nella massa i valori di forza, energia, durezza, fierezza, patria, militarismo, senso del dovere e fede nell'ideale del fascismo.

\section{TURBINE D’AMORE}

Mussolini a cavallo piaceva alle donne: lo abbiamo visto con Claretta Petacci e Wera B.M.; però chi si esprimeva in termini amorosi verso il condottiero del fascismo erano ancora di più gli uomini. Ecco cosa si legge ancora nel romanzo di Mario Carli quando il protagonista Falco partecipa alle celebrazioni bolognesi dell'anniversario della Marcia su Roma culminate nell'inaugurazione dello Stadio Littoriale:

le giornate bolognesi lo presero nel loro composto delirio, lo travolsero nella frenesia moltitudinaria dell'amore suscitato dall'apparizione del Duce guerriero, alto a cavallo sull'oceano di baionette che nel Littoriale lo acclamavano [...]; egli [Falco] fu la molecola vibrante in quel turbine d'amore, e per quattro giorni non vide che il suo Duce ${ }^{32}$.

C'è un evidente omoerotismo in questa descrizione: Falco e gli altri che sono presi nel "turbine d'amore" e nella "frenesia [...] dell'amore suscitato dall'apparizione del Duce guerriero, alto a cavallo" sono uomini. Abbiamo visto su quali presupposti teorici poggiava una descrizione come questa di Carli: seguendo Le Bon non era difficile visualizzare il rapporto tra il Duce e la folla in termini amorosi. Inoltre - non dimentichiamolo - qui ci troviamo di fronte a un "oceano di baionette", cioè a una folla di militari e combattenti, e questo riporta tutto entro i confini di quella che Lorenzo Benadusi ha chiamato

32. Carli Mario, L'Italiano di Mussolini, cit., pp. 195-196. 
"rispettabilità in uniforme", per cui anche il sottotesto omoerotico poteva venire facilmente accettato ${ }^{33}$.

E chi non si riconosceva in quell'immagine e nel tipo di relazione a cui essa chiamava? Abbiamo visto che Gadda, caduto il regime, sceglie la strada dell'invettiva, scagliandosi contro "la priapata a cavallo del Gran Pernacchio"34 e contro l'Eros che legava il Duce alle masse. Ma il caso di Gadda è complesso, perché il suo rapporto con il fascismo non è stato lineare. Gadda, reduce del primo conflitto mondiale, aveva aderito al fascismo nella speranza di sciogliere il nodo creato dalla Grande Guerra; crollata questa speranza, a un "riposizionamento $[\ldots]$ in un'area antimussoliniana" non gli bastano, come ha scritto Cristina Savettieri, "l'uso delle categorie della psicanalisi e la forma dell'invettiva ad personam": "Eros e Priapo rimane inchiodato al soggetto che parla, che vuole accusare, accusarsi e discolparsi insieme, nel tentativo disperato di allontanarsi dalla storia" 35 .

Diversa è la strada imboccata da Palazzeschi, che era anti-interventista al momento della prima guerra mondiale e che non ha mai simpatizzato né aderito al fascismo. Con lui però bisogna cominciare da ancor più lontano.

\section{CAVALIERE BIANCO}

Ben prima che il Duce si proponesse come il Condottiero d'Italia la fantasia di Palazzeschi ventenne era stata colpita da un altro cavaliere che si aggirava per le colline di Settignano vicino a Firenze:

Nell'estate del 1898 in questo paese [...] apparve un uomo a cavallo, seguito sempre da un levriero elegante ed agilissimo. Era vestito di bianco

33. Cfr. Benadusi Lorenzo, Il nemico dell'uomo nuovo. L'omosessualità nell'esperimento totalitario fascista, prefazione di Emilio Gentile, Milano, Feltrinelli, 2005, p. 281: "Paradossalmente [...] proprio la massima esaltazione della virilità e dello stile guerresco, il raggiungimento dell'uomo collettivo organizzato, del cittadino-soldato e di una 'rispettabilità in uniforme', militare e bellicosa, facevano diminuire l'ostracismo nei confronti degli omosessuali".

34. Gadda Carlo Emilio, Eros e Priapo, cit., p. 20.

35. SAVETTIERI Cristina, "Il ventennio di Gadda", in Scrittori italiani tra fascismo e antifascismo, a cura di Romano Luperini e Pietro Cataldi, Pacini, Pisa, 2009, p. 32. Cfr. anche Robert S. Dombroski, "Gadda e il fascismo", in Gadda e il barocco, traduzione di A.R. Dicuonzo, Torino, Bollati Boringheri, 2002, p. 137: in Eros e Priapo "l'invettiva contro l'ipertrofia dell'io e le sue manie di grandezza agisce da schermo [...] come una sorta di esorcismo, come una satira diretta contro l'io, contro la tendenza dell'io vittimista [...] a ostentare [...] la propria solitudine e, nel far ciò, a rivalersi sulla storia". 
e portava il cappello a grande tesa. Non andava che a cavallo inoltrandosi per stradine impervie, [...] chiamando ogni tanto il bel levriero, Sirio, per frenarne la nobile impazienza e l'avventuroso ardimento, ma non con voce di padrone autoritaria, calda bensì, come quella di un amico. Nessuno sapeva allora chi fosse quell'uomo con precisione: cane cavallo e cavaliere avevano qualcosa che li distanziava ed isolava nel modo più assoluto dal mondo circostante $[\ldots]^{36}$.

"Il cavaliere bianco era un poeta dal nome profetico": d'Annunzio, il Vate, riconoscibilissimo in tutte le sue notissime pose ed eccentricità. Palazzeschi è bene attento a non nominarlo mai direttamente, acuendone così il carattere irraggiungibile e facendolo diventare come il "personaggio di una favola" 37 . Elegante, dall'aria nobile, questo principe favoloso gira sempre a cavallo accompagnato da un aristocratico levriero, Sirio (lui sì, nominato), il cui nome così ricercato distanzia ancor più cane e padrone dai comuni mortali, proiettandoli lontano dalla Terra, verso gli astri.

Palazzeschi nota anche la voce del misterioso cavaliere: è calda, "come quella di un amico", tutto fuorché autoritaria. È una caratteristica che risalta particolarmente in questa figura distante e aristocratica, che facilmente immagineremmo algida e che invece si circonfonde per un attimo di una nota di calore, di un tratto amichevole. È l'unica concessione umana fatta a questo "uomo, sempre a cavallo, [che] si poteva vedere ogni giorno a tale altezza" 38 , sopra tutti gli altri uomini.

Anche questo cavaliere ha i suoi amori: ed è seguendoli nella loro vicenda che Palazzeschi disegna la parabola discendente dell'astro della Capponcina dalla favola alla farsa boccaccesca. Dapprima è "un'attrice celeberrima”, Eleonora Duse, "invisibile in modo assoluto" e anche lei mai nominata. Dileguatasi "la Signora", inizia una carovana di "donne visibilissime [...]: marchese, contesse, principesse, taluna amazzone provetta che tutti potevano ammirare cavalcando in pieno sole al fianco del cavaliere [...]. E cantanti altresì, delle grandi come delle piccole scene, ballerine e donnine del varietà" 39 . La rottura con la grande attrice tragica segna l'inizio di una discesa del cavaliere; tale discesa, però, non

36. Palazzeschi Aldo, "Un uomo a cavallo" [1958], in Il piacere della memoria, Milano, Mondadori, 1964, p. 545. Il pezzo era uscito sul Corriere d'Informazione il 25 febbraio 1958, come si ricava dalla Bibliografia degli scritti di Aldo Palazzeschi, a cura di Anna Grazia D’Oria, Roma, Edizioni di Storia e Letteratura, 1982, pp. 64 e 74.

37. Ibid., p. 546.

38. Ibid.

39. Ibid., pp. 546-547. 
ha nulla di drammatico, bensì si svolge all'insegna di una sproporzione sottilmente comica:

da quel giorno le consuetudini del cavaliere bianco subirono fondamentali cambiamenti di proporzioni e di rotta [...]. Non si vedeva più vagare solitario seguito dall'aristocratico levriero, ma era seguito sempre da staffieri scudieri palafrenieri $[\ldots]$ e da un nugolo di levrieri che alle gambe dei cavalli conformavano con disciplina la loro gamba. Era oramai una corte e passava simile a quelle che si potevano ammirare al tempo di Lorenzo de' Medici, di Can Grande della Scala, degli Sforza, degli Estensi e dei Gonzaga ${ }^{40}$.

Finché, rovinato dai debiti, "Il cavaliere bianco, non si sa se a cavallo o in ferrovia, per un lontano lido disparve" e le innumerevoli e più varie suppellettili che adornavano la sua dimora vennero messe all'asta: tra queste Palazzeschi osserva "un curioso recipiente", "l'ultimo dei misteri" ", di cui viene taciuta ma si intuisce la vera natura e funzione - si tratta di un pitale, di un orinale -; e così attraverso questa ulteriore mancata nominazione vengono accomunati per un attimo in chiusura il nome proprio dell'alto poeta e il nome comune dell'oggetto più impoetico.

\section{Cavaliere Nero}

Una ventina di anni più tardi un altro personaggio si impone all'attenzione degli italiani: Mussolini, nerovestito, aggressivo, con una "voce alta e roca, tra quella del banditore delle piazze e l'attore delle arene" ${ }^{\text {"42. }}$. In occasione dell'inaugurazione di via dell'Impero a Roma, avvenuta nel 1932, Palazzeschi lo descrive così:

Era a cavallo $[\ldots]$ con l'elmo e la spada. Un elmo d'acciaio alla cui sinistra s'innalzava il pennacchio nel cielo simile allo spillo delle fontane in giornate di festa e la spada, alta nella mano, vi produceva scintille. E l'elmo era così formidabile e incapato bene che solo la bocca lasciava scoperta del Duce, la quale congiungendosi all'arricciatura posteriore gli conferiva, sotto la cupola, una bocca circolare ${ }^{43}$.

40. Ibid.

41. Ibid., p. 549.

42. Palazzeschi Aldo, Tre imperi... mancati. Cronaca 1922-1945 [1945], a cura di Gino Tellini, Milano, Mondadori, 2016, p. 6. Cfr. anche p. 51 ("voce roca e possente").

43. Ibid., p. 11. 
In realtà per l'inaugurazione di via dell'Impero Mussolini non indossava l'elmo d'acciaio e non portava la spada: aveva sì il pennacchio ma su un copricapo era di tela, e per tagliare il nastro inaugurale della nuova arteria stradale usò un paio di forbici ${ }^{44}$. La descrizione di Palazzeschi mette insieme diverse immagini del Duce a cavallo: quella con l'elmo d'acciaio, che il Duce portava in altre occasioni e che effettivamente indossò cavalcando sulla via dell'Impero circa un anno e mezzo dopo, per celebrare l'ottava leva fascista nel maggio del $1934^{45}$; e quella con la spada dell'Islam, brandita in alto verso il cielo di Tripoli nel $1937^{46}$. La commistione palazzeschiana, se pure non rispetta la realtà storica, è però funzionale all'intento dell'autore: fare un ritratto grottesco del Capo del fascismo. Come ha scritto Gadda, Palazzeschi "ci dà la cronaca delle deformazioni strutturali che i fatti e le persone del dramma - e sopra tutti il pupazzo numero uno, il laido protagonista della tragica farsa - hanno via via subito nella sua percezione" ${ }^{\prime 7}$.

Qui Mussolini ha un "pennacchio" che s'innalza nel cielo "simile allo spillo delle fontane in giornate di festa". La spada produce scintille: come fosse un fuoco d'artificio. Ma il particolare più incisivo è quell'elmo che è come una cupola ed è così bene infilato sopra la testa che - immagina Palazzeschi - copre occhi e naso e lascia libera solo la bocca. Siamo nel comico, ma solo per scivolare immediatamente nella deformazione grottesca: quella bocca, allargandosi e congiungendosi all'arricciatura posteriore dell'elmo, diventa circolare, a trecentosessanta gradi. Viene in mente la scultura Profilo continuo. Dux (1933-35) di Renato Bertelli ${ }^{48}$, artista toscano come Palazzeschi: soltanto che qui invece di un elegante dinamismo futurista abbiamo uno slabbrarsi all'indietro: quella bocca dalla quale uscivano "siluri, $[\ldots]$, granate, $[\ldots]$ colpi di cannone" e che sparava "bombe" 49 in eccesso su tutta la linea, è circolare come l'elmo, si apre da ogni lato, come la brama di potere e di protagonismo del Duce.

44. Cfr. la copertina dell'Illustrazione italiana, anno LIX, n. 45, 6 novembre 1932.

45. Cfr. Giornale LUCE B0476 del 05/1934 visibile sul sito dell'Archivio LUCE www.archivioluce.com.

46. Cfr. la fotografia A00071465 scattata a Tripoli il 18-03-1937 visibile sul sito dell'Archivio LUCE www.archivioluce.com

47. GADDA Carlo Emilio, "I tre imperi", in Il Mondo, II, 30, Firenze, 15 giugno 1946, ora in ID., Saggi giornali favole e altri scritti, I, a cura di Liliana Orlando, Clelia Martignoni, Dante Isella, Milano, Garzanti, 1991, p. 941.

48. Cfr. Novecento. Arte e vita in Italia tra le due guerre, catalogo della mostra (Forlì, 2 febbraio - 16 giugno 2013), a cura di Fernando Mazzocca, Cinisello Balsamo (Milano), Silvana, 2013, p. 141.

49. Palazzeschi Aldo, Tre imperi... mancati, cit, p. 78. 
La caricatura della rappresentazione non si ferma qui ma prosegue insieme al racconto:

Via via che procedeva fra milizie stecchite e selve di baionette, il pennacchio andava sempre più in su e la spada, contro il sole, produceva sempre più rapide scintille. Squarci di trombe laceranti, cannonate, inni, canti, invocazioni, mentre sulla via dell'Impero procedeva il Duce [...] alto sulle quadrate legioni; non più fiero né feroce né terribile, ma enorme. [...] Mise le ali il suo cavallo bianco su quel tappeto impalpabile [della via dei Trionfi], e la bocca circolare sembrava dilatarsi sotto l'elmo a ondate sempre più vaste, fino a lambire l'orizzonte ${ }^{50}$.

Il pennacchio sale sempre più in alto e la spada manda sempre più scintille: dietro la maestosità solo apparente di questo incremento si intuisce un baraccone da fiera. L'accompagnamento sonoro gioca sulla stessa sottile ambiguità. Alla fine il Duce non appare più feroce ma soltanto "enorme", smisurato come i suoi proclami e le sue promesse. Finché la rappresentazione sfocia nel fantastico: il cavallo bianco è come se volasse e la bocca del cavaliere, da sotto l'elmo incapato bene, pare che si dilati a dismisura, si allarghi fino a toccare l'orizzonte ${ }^{51}$.

C’è una qualche attrattiva erotica in questo cavaliere? Nessuna: Palazzeschi innalzando il pennacchio, incrementando le scintille della spada, dilatando la bocca, ne ha aumentato i contorni e le proporzioni ma lo ha svuotato di ogni charme, se mai ne era stato provvisto. Fin dall'inizio, da quando il Duce si affaccia al balcone, lo scrittore fiorentino lo riduce infatti a un pacchiano parvenu che ha del "carrettiere siciliano", del "toreador" e del "vecchio Lord"52 insieme.

Nel rapporto tra il Capo e la folla descritto da Palazzeschi non c’è tanto la presenza di Eros quanto la mancanza, la cancellazione di Logos. In particolare la donna, più che mossa dall'inconscio, è ridotta a una funzione biologica riproduttiva, ad animale; ma è il Duce stesso che la riduce a questo:

Per quello che riguardava le donne si mostrò poco esigente con esse: di cervello non ne parlò neppure; non avrebbero avuto né un viso, né un abito né un

50. Ibid., pp. 12-13.

51. Cfr. ancora GADDA Carlo Emilio, "I tre imperi”, cit., p. 940: "Le grandi manovre e le grandi parate della bestiaggine lo [Palazzeschi] vedono abbrividire, inorridire: muto e sconvolto presenzia a funeralesca maccheronata, ben comprendendo che a quei maccheroni e a quei pennacchi, a quei neri fiocchi, terranno dietro l'abiezione e la fame, il galoppo finale della distruzione e il trionfo della morte. [...] Non facile accompagnare il dolore mero e la sbigottita speranza per mezzo la sarabanda feroce, fra i coriandoli e le strombettate della stoltezza".

52. Palazzeschi Aldo, Tre imperi... mancati, cit., p. 5. 
nome, ma quella sola parte che per essere celata bene non è discreto nominare. Le donne si sentirono, a queste parole, delle arpe a cui era rimasta una corda sola: la centrale. [...] E, cosa in sommo grado stupefacente, a nessuna venne fatto di pensare alle gatte o alle cagne, alle scrofe, alle vacche o alle coniglie: il cervello aveva già preso la posizione orizzontale. Che cosa vuol dire la forza di un genio creatore. E fu questo il primo miracolo del Duce. ${ }^{53}$

Il gioco di seduzione tra Mussolini e le donne si riduce, nel ritratto palazzeschiano, a un monotono meccanismo dove alle donne è dato soltanto, oltre che fare figli per la patria, lodare continuamente la bellezza del Capo:

È certo che il Duce dovette ritenersi molto bello, bellissimo, di una bellezza indiscutibile, quasi classica, tenuto conto dell'elasticità di tale attribuzione; glie lo avevano detto le donne in tutte le maniere $[\ldots] .^{54}$

Non si vede nessuna interazione, nessun movimento di conquista, nessun senso di scoperta in questi scambi. In Gadda le donne "perdono [...] le staffe" ma almeno lui "le isguarda e le concupisce". Qui il gioco si riduce a una ubiqua, univoca ecolalìa femminile a lode del Capo.

Se il corpo femminile vale solo come macchina per figliare e quello maschile deve avere per unico modello quello del Duce ("Un torace massiccio su corte ed esili gambe" lo descrive Palazzeschi ${ }^{55}$ ), cosa rimane del desiderio e dei piaceri della seduzione? Dopo i fasti dannunziani della Belle Époque dov'è finito Eros? Lo smisurato cavaliere nero, con "quella voce che faceva accapponare la pelle", "una voce che terrorizzava" 56 , non ha nulla di paragonabile all'elegante e irripetibile cavaliere bianco dalla voce "calda [...] come quella di un amico". Dove ritrovare allora un principio di piacere? A cavallo, lo abbiamo visto sopra, il piacere si trova ancora nei "ragazzoni sicuri, virili", dalle "coscie enormi, massicce come colonne", che sfilano sotto le finestre delle Materassi creando loro indicibili turbamenti. Altrimenti in dosi ben più massicce e ingestibili, senza bisogno di cavalcatura, Eros esplode in casa Materassi "con lo scultoreo Remo che" - come ha scritto Francesca Serra - "fa da sole a cui ciascuno, uomo o

53. Ibid. pp. 8-9.

54. Cfr. ibid. pp. 15-16.

55. Cfr. ibid., p. 5. Cfr. anche GADDA Carlo Emilio, "I tre imperi", cit., p. 940: "Io sono un maschio!...': e che ce ne importa? Maschio o femmina, era un rospaccio con le gambe a roncola".

56. Palazzeschi Aldo, Tre imperi... mancati, cit., pp. 79 e 100. 
donna senza distinzione, è chiamato a rendere il suo tributo libidinale"57. Remo, motore incontrollato di piacere, permette a Palazzeschi di esprimere attraverso lo sguardo di due vecchie zitelle desideri altrimenti inconfessabili ${ }^{58}$; ma non solo: anche di spostare il luogo dell'Eros rimuovendolo dai canali obbligati del discorso pubblico fascista allora dominante.

\section{GiovanotTo IN MUTANDINE}

Nato nella finzione narrativa nel 1905, praticamente quando era appena terminato il legame tra d'Annunzio e Duse, Remo irrompe nella vita delle ricamatrici in quella Settignano dove il Vate a cavallo era stato come il principe delle favole. Risucchiate le energie libidinali così come le finanze delle zie Teresa e Carolina e della serva Niobe, il ragazzo infine si sposa ventiquattrenne e si trasferisce in America. L'epilogo della vicenda, con Remo lontano e le vecchie Materassi ridotte in miseria a cucire per le popolane un tempo sdegnate, si riallaccia più o meno al periodo in cui fu iniziato il romanzo, nel $1932^{59}$. Nell'anno del decennale fascista, quando il Duce inaugurava via dell'Impero col suo pennacchio sempre più alto e la sua bocca sempre più larga, Palazzeschi cominciava a dar corpo a una vicenda dove il protagonista è un giovane semidio che sommuove $\mathrm{i}$ desideri più incontrollabili e indicibili, campione di una virilità di segno opposto a quella mussoliniana ${ }^{60}$. Il suo corpo, a differenza di quello del Duce (del Duce come lo vedeva Palazzeschi), suscita continuamente desiderio; anche quando non è più presente in carne e ossa: anche in fotografia, come in quella che, ingrandita, campeggia alla fine del romanzo nel laboratorio di Teresa e Carolina:

Remo era solo e nudo con delle mutandine da bagno piuttosto piccole e succinte, per modo che ogni efficienza del corpo vi risultava in tutto il suo splendore. La fotografia era stata fatta sul limitare dell'acqua. Il giovane era a testa alta davanti al sole la cui vivida luce non giungeva a turbarne la serenità del viso, ma gli conferiva un leggerissimo accigliamento, un lieve cruccio che gli stava tanto bene. Aveva dietro, per isfondo, il mare ${ }^{61}$.

57. Serra Francesca, "Introduzione" in Palazzeschi Aldo, Sorelle Materassi, cit., p. XIX.

58. Cfr. ibid.

59. Cfr. ibid., pp. X-XI.

60. Francesca Serra nota che "Virile' [...] è il refrain, la parola-chiave impiegata dal narratore per seguire con amorevole attenzione la crescita e l'irrobustirsi del gagliardo" Remo (ibid., p. XVI).

61. Palazzeschi Aldo, Sorelle Materassi, cit., pp. 300-301. 
In confronto a questa che giganteggia nel laboratorio delle ricamatrici, le innumerevoli foto del Duce diffuse dalla propaganda scompaiono ${ }^{62}$. Neppure quando per un attimo Palazzeschi ci lascia immaginare il corpo nudo del dittatore in fotografia si sentono i brividi della carne: "per chi lo voleva nudo... c'era anche quello, su questa terra cosa non c'è?"'63; è soltanto l'ennesima posa di fronte all'obiettivo fotografico usato fin troppo largamente.

Negando ogni tipo di fascino al corpo del dittatore e spostando tutte le attrattive sulle fattezze del giovane Remo, minuziosamente descritto e costantemente ammirato, Palazzeschi riesce non soltanto a far passare la desiderabilità di un corpo maschile in una pulsione omosessuale, ma anche a prendere sornionamente le distanze dalla virilità fascista. Lo fa senza darlo a vedere; anzi, supremo sberleffo, facendola apparire brevemente nelle ultimissime pagine - dopo che del fascismo in Sorelle Materassi non si è vista neanche l'ombra - in un confronto impari tutto a favore di Remo:

Le contadine ammiravano il ritratto senza riserve [...]. Facevano i confronti coi figli loro: "il milite, l'avanguardista, il premilitare, il calcista, il corridore, il pugile...". Anche loro erano sempre in mutandine: "anche il mio, come il mio, anche il mio è fatto così, ha certe spalle! Se vedeste che gambe! [...]"

Le sorelle [Materassi] sorridevano concedendo alla clientela tutti i confronti, ma la sapevano lunga, e quando erano sole rimettevano al posto le cose:

"Poverette, sono i loro figlioli [...], non gli si può dir di no"

[...] Per quanto potessero essere belli e forti i loro figlioli quello era eccezionale, fuori discussione.

E nessuno si faceva caso di trovare in quella stanza il ritratto di un giovanotto in mutandine $e^{64}$.

62. Cfr. Palazzeschi Aldo, Tre imperi... mancati, cit., pp. 14-15: "Avrebbe potuto lasciarsi fotografare $[. .$.$] una volta per tutte, e imporre quel ritratto [...]. Invece no$ [...], magnanimo, volle che ogni desiderio fosse soddisfatto [...]. C'era chi lo voleva in borghese e chi in uniforme $[\ldots]$. Chi accigliato $[\ldots]$ e chi sorridente $[\ldots]$. Chi col cappello e chi con la pelata [...]. A piedi e a cavallo, in aeroplano e in motocicletta; in motoscafo, in barca, ritto e a sedere; al volante; solo o in gruppo con ogni specie di persone [...]. I collezionisti delle sue fotografie possono testimoniarlo".

63. Ibid., p. 15. Né maggiore attrattiva avevano per Palazzeschi le foto di Mussolini al mare (a differenza di quella romantica di Remo): "a galla frangendo a nuoto le onde o [...] sulla linea del bagnasciuga nell'ora del crepuscolo e con un movimento delle braccia eccessivamente marcato" (ibid.).

64. Palazzeschi Aldo, Sorelle Materassi, cit., pp. 317-319. 
Nessuna delle massaie fasciste, nuove clienti delle Materassi, e nessuno dei lettori. Nella piccola Settignano, dove un tempo si aggirava un cavaliere "vestito di bianco" e si favoleggiava dei suoi amori, ora "un ingrandimento, due terzi circa della grandezza naturale" 65 , mostra impunemente il corpo divino di un giovane "solo e nudo con delle mutandine da bagno piuttosto piccole e succinte", ammirato da tutti, mentre a Roma il Duce cavalca su donne e uomini imbrigliati e docili come Ned e gli altri cavalli della sua scuderia.

Beatrice SICA

University College London 
